\title{
Truncating the memory time in nonequilibrium dynamical mean field theory calculations
}

\author{
Michael Schüler, ${ }^{1}$ Martin Eckstein, ${ }^{2}$ and Philipp Werner ${ }^{1}$ \\ ${ }^{1}$ Department of Physics, University of Fribourg, 1700 Fribourg, Switzerland \\ ${ }^{2}$ Department of Physics, University of Erlangen-Nürnberg, 91058 Erlangen, Germany
}

(Received 25 April 2018; published 19 June 2018)

\begin{abstract}
The nonequilibrium Green's functions (NEGF) approach is a versatile theoretical tool, which allows to describe the electronic structure, spectroscopy, and dynamics of strongly correlated systems. The applicability of this method is, however, limited by its considerable computational cost. Due to the treatment of the full two-time dependence of the NEGF, the underlying equations of motion involve a long-lasting non-Markovian memory kernel that results in at least an $N_{t}^{3}$ scaling in the number of time points $N_{t}$. The system's memory time is, however, reduced in the presence of a thermalizing bath. In particular, dynamical mean field theory (DMFT), one of the most successful approaches to strongly correlated lattice systems, maps extended systems to an effective impurity coupled to a bath. In this work, we systematically investigate how the memory time can be truncated in nonequilibrium DMFT simulations of the Hubbard and Hubbard-Holstein models. We show that suitable truncation schemes, which substantially reduce the computational cost, result in excellent approximations to the full time evolution. This approach enables the propagation to longer times, making fundamental processes such as prethermalization and the final stages of thermalization accessible to nonequilibrium DMFT.
\end{abstract}

DOI: 10.1103/PhysRevB.97.245129

\section{INTRODUCTION}

The theoretical study of nonequilibrium phenomena in correlated lattice systems is an active field of research, which is driven by the rapid development of ultrafast laser techniques and remarkable experimental discoveries in light-driven materials [1-5]. One of the challenges in the numerical simulation of the dynamics after a photoexcitation or parameter quench is the emergence of different relevant timescales (Fig. 1). In lattice systems, the excitation process typically happens on the timescale of the inverse electron hopping, corresponding to femtoseconds in correlated electron materials [6,7]. If the driving laser field contains several cycles, the system may be transiently described by a so-called Floquet state, which can exhibit properties quite different from the equilibrium states of the initial Hamiltonian. After the pulse, a relaxation process sets in, which eventually results in a new thermal state. This relaxation may involve the transient trapping in long-lived prethermalized [8-12] or prerelaxed [13] states, in which local observables look thermalized, while nonlocal ones are not, or the passage near nonthermal critical points $[14,15]$. In the case of integrable isolated quantum systems, the prethermal state can be described by a generalized Gibbs ensemble (GGE) [16,17]. The coupling to slow degrees of freedom such as phonons can introduce additional timescales in the thermalization process [18-21].

Developing analytical or numerical methods that can bridge these different timescales and describe the evolution of the system from the initial excitation process to the final, thermalized equilibrium state is a major challenge. While numerical approaches such as time-dependent exact diagonalization $[18,22]$, density-matrix renormalization group methods [23], or the nonequilibrium Green's functions (NEGF) approach $[24,25]$ accurately capture the short-time evolution during the excitation and initial relaxation process, a quantum kinetic description such as the generalized Kadanoff-Baym ansatz (GKBA) [26-29] or related methods [30-32] allow to approximately describe the thermalization dynamics. In particular, the GKBA assumes a decoupling of the spectral properties (encoded in the two-time spectral function) and the occupation dynamics described by the single-particle reduced density matrix. One possible strategy is the development of a multiscale approach, where the photoexcited charge carrier distribution obtained by an accurate method is used to initialize a kinetic equation [33]. However, it is not clear whether the $a d$ hoc approximations entailed by such an approach compromise the long-time dynamics. This issue is particularly relevant for systems with long-range order, where a fully self-consistent treatment is crucial for the dynamics of the order parameter. For these reasons, a formalism which allows to treat the entire evolution and the relevant physical processes within a numerically efficient, consistent, and controllable scheme would be highly desired.

An approximate method which can, in principle, capture the different stages in the time evolution of a photoexcited correlated lattice model is the dynamical mean field theory (DMFT) [34-36]. It maps the lattice system onto a self-consistently determined quantum impurity model, and is formulated directly in the thermodynamic limit. While the basic approximation of this scheme, the local nature of the self-energy [37,38], neglects processes that are related to nonlocal fluctuations and correlations, it can describe the excitation by strong laser fields $[36,39,40]$, the trapping in prethermalized states $[10,14]$, and the relaxation into a new thermal equilibrium state [40,41]. Furthermore, the method can be systematically extended to include nonlocal effects: cluster versions of DMFT [42-44] allow to capture the effect of short-range correlations, while extended DMFT (EDMFT) [45,46] and combinations with the $G W$ approximation ( $G W+$ EDMFT) allow to incorporate local and nonlocal polarization effects $[47,48]$. This versatility 


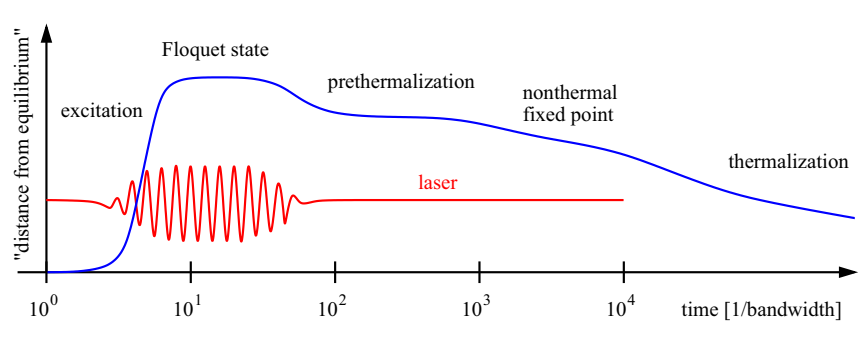

FIG. 1. Schematic illustration of the different timescales appearing in the evolution of a photoexcited correlated electron system. (Adapted from Ref. [34].)

makes the nonequilibrium DMFT one of the currently most powerful techniques for the study of photoexcited lattice models and, in particular, strongly correlated systems.

A crucial step in this NEGF-based technique is the solution of the two-time equation of motion for the single-particle Green's function (GF), which defines an integrodifferential equation on the Keldysh [Fig. 2(a)] or Kadanoff-Baym [Fig. 2(b)] contour. Many-body effects are captured by the two-time self-energy, which plays the role of a memory kernel for the time evolution. Even if a numerically cheap approximate impurity solver is employed, nonequilibrium DMFT calculations implemented with a fixed discretization of the time contour scale at least cubically in the number of time steps $N_{t}$. (If a higher-order perturbative impurity solver is employed, the numerical effort scales with a correspondingly higher power of $N_{t}[49,50]$.) This high computational cost originates from the fact that the entire memory of the previous time evolution is kept in the calculation of the interacting GF, which limits in practice the maximum simulation times and thus the parameter regimes in which the full time evolution from excitation to thermalization can be studied.

A very simple idea to overcome this limitation is to truncate the memory time of the self-energy kernels. This approach roots in the effective reduction of the system's memory when it is coupled to a bath with broad spectrum, which is also the basis for Markovian approximations in open quantum systems [51,52]. In the context of DMFT, the mapping of the correlated electrons on a lattice to an effective impurity model coupled to a (self-consistently determined) bath should naturally result in a decay of the memory kernel. Hence, a properly implemented truncation scheme is expected to have a small effect on the accuracy of the results. By introducing a variable cutoff

(a)

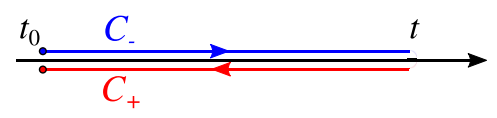

(b)

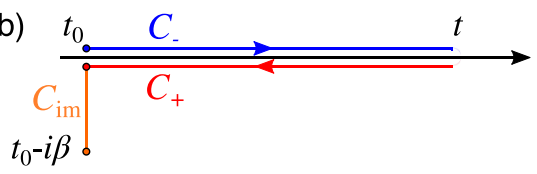

FIG. 2. The time arguments of the Green's functions lie on the contour $\mathcal{C}$ consisting of the forward $\left(\mathcal{C}_{-}\right)$and backward $\left(\mathcal{C}_{+}\right)$branches along the real axis, and the imaginary branch $\mathcal{C}_{\text {im }}$. The arrows indicate the direction of the contour ordering. $\beta$ denotes the inverse temperature. parameter, such an approach also allows to systematically check and control the effect of the approximation. A truncation of the memory time reduces the numerical cost of the Dyson equation and related convolution integrals by at least one order, which should enable the study of long-time dynamics without resorting to multiscale approaches or uncontrolled approximations.

Motivated by this perspective, we investigate the effect of memory truncations in nonequilibrium DMFT. In particular, we demonstrate the feasibility of the approach for paradigmatic examples: the Hubbard model in the paramagnetic and antiferromagnetic (AFM) phase, as well as for the Hubbard-Holstein model. The formalism is introduced and explained in Sec. II, while an analysis of the nonequilibrium dynamics of these models for different memory times is presented and discussed in Sec. III. Conclusions are presented in Sec. IV.

\section{FORMALISM}

We focus our analysis on the Hubbard-Holstein model, given by the Hamiltonian

$$
\begin{aligned}
\hat{H}= & -v \sum_{\langle i j\rangle, \sigma} \hat{c}_{i \sigma}^{\dagger} \hat{c}_{j \sigma}+\frac{U}{2} \sum_{i, \sigma} \hat{n}_{i \sigma} \hat{n}_{i \bar{\sigma}} \\
& +g \sum_{i, \sigma} \hat{n}_{i \sigma}\left(\hat{b}_{i}+\hat{b}_{i}^{\dagger}\right)+\omega_{\mathrm{ph}} \sum_{i} \hat{b}_{i}^{\dagger} \hat{b}_{i} .
\end{aligned}
$$

The first term describes the nearest-neighbor hopping of electrons with spin $\sigma$ (fermionic creation and annihilation operators $\hat{c}_{i \sigma}^{\dagger}$ and $\hat{c}_{i \sigma}$, respectively) with amplitude $v$, the second term the onsite Coulomb repulsion of electrons with different spin, parametrized by $U$, while the third term represents the Holstein-type electron-phonon (e-ph) coupling (bosonic operators $\hat{b}_{i}$ ). The last term is the phonon Hamiltonian. In what follows, we measure energies in units of the quarter bandwidth $w$ and time in units of $1 / w$.

The central quantity in a NEGF-based treatment of the Hamiltonian (1) is the single-particle lattice GF in real space

$$
G_{i j, \sigma}\left(t, t^{\prime}\right)=-i\left\langle\mathcal{T} \hat{c}_{i \sigma}(t) \hat{c}_{j \sigma}^{\dagger}\left(t^{\prime}\right)\right\rangle
$$

or in momentum space

$$
G_{\mathbf{k}, \sigma}\left(t, t^{\prime}\right)=-i\left\langle\mathcal{T} \hat{c}_{\mathbf{k} \sigma}(t) \hat{c}_{\mathbf{k} \sigma}^{\dagger}\left(t^{\prime}\right)\right\rangle .
$$

The time arguments of the GF are located on the KadanoffBaym contour [Fig. 2(b)], while $\mathcal{T}$ denotes the corresponding contour-ordering operator. The operators and, accordingly, the GF in momentum space are obtained by Fourier transformation with respect to the underlying lattice. The latter also defines the free-electron dispersion $\varepsilon_{\mathbf{k}}$, which corresponds to the eigenvalues of the hopping matrix in Eq. (1).

The lattice GF (3) obeys the Kadanoff-Baym equation (KBE)

$$
\left(i \partial_{t}+\mu-\varepsilon_{\mathbf{k}}\right) G_{\mathbf{k} \sigma}\left(t, t^{\prime}\right)-\int_{\mathcal{C}} d \bar{t} \Sigma_{\mathbf{k} \sigma}(t, \bar{t}) G_{\mathbf{k} \sigma}\left(\bar{t}, t^{\prime}\right)=\delta_{C}\left(t, t^{\prime}\right) .
$$

Here, $\mu$ stands for the chemical potential and the self-energy $\Sigma_{\mathbf{k} \sigma}\left(t, t^{\prime}\right)$ (which is a functional of the GF) captures all 
many-body effects. $\delta_{C}\left(t, t^{\prime}\right)$ denotes the Dirac delta function with respect to contour arguments.

While the following analysis is carried out for the Hubbard and Hubbard-Holstein models, we stress that the statements on the effects of truncations of the memory time are generically valid. They also apply to DMFT simulations of multiband models and extended DMFT formalisms.

\section{A. Dynamical mean field theory in the strong-coupling limit}

DMFT maps a correlated lattice model [the Hamiltonian (1) in our case] to a quantum impurity model with a selfconsistently determined bath. The main approximation is the assumption of a spatially local self-energy, implying $\Sigma_{\mathbf{k} \sigma}\left(t, t^{\prime}\right) \approx \Sigma_{\sigma}\left(t, t^{\prime}\right)$. This approximation becomes exact in infinite-dimensional systems [37,38]. The local self-energy $\Sigma_{\sigma}\left(t, t^{\prime}\right)$ can be computed from the solution of a suitably defined auxiliary impurity system. Given a self-energy, the lattice KBE (4) can be solved to obtain the approximate DMFT lattice GF. The self-consistent solution is constructed such that the local lattice GF,

$$
G_{\mathrm{loc}, \sigma}\left(t, t^{\prime}\right)=\frac{1}{V_{\mathrm{BZ}}} \int_{\mathrm{BZ}} d \mathbf{k} G_{\mathbf{k} \sigma}\left(t, t^{\prime}\right),
$$

is identical to the impurity Green's function $G_{\mathrm{imp}, \sigma}\left(t, t^{\prime}\right)$. Here, $\mathrm{BZ}$ stands for the Brillouin zone and $V_{\mathrm{BZ}}$ the corresponding volume.

Different methods can be employed to solve the impurity problem [34]. For the nonequilibrium scenarios that we consider in this study, suitable methods for strong electronelectron interaction are strong-coupling perturbative methods [49] such as the noncrossing approximation (NCA) [53] or one-crossing approximation (OCA) [54]. Here, the impurity problem is treated by solving the local many-body problem exactly (energies $E_{\alpha}$ ), which serves as a reference, while the hopping from and to the surrounding bath is captured by the hybridization function $\Lambda_{\sigma}\left(t, t^{\prime}\right)$ (which is similar to an embedding self-energy). Defining the so-called pseudoparticle GF $\mathcal{G}_{\alpha}\left(t, t^{\prime}\right)$ ( $\alpha$ labels the local many-body states) as a correlator of the local many-body operators, the impurity problem can be treated by diagrammatic methods. In this case, the pseudoparticle selfenergy $\Sigma_{\alpha}\left(t, t^{\prime}\right)$ becomes a functional of $\Lambda_{\sigma}\left(t, t^{\prime}\right)$ and $\mathcal{G}_{\alpha}\left(t, t^{\prime}\right)$. Expanding $\Sigma_{\alpha}\left(t, t^{\prime}\right)$ in powers of the hybridization function, the NCA corresponds to the first-order approximation beyond the atomic limit, while OCA corresponds to the second-order scheme.

The impurity KBE for the pseudoparticle GF $\mathcal{G}_{\alpha}$ is similar to Eq. (4). However, the convolution integral has a slightly different form: the integrand is nonzero only if the times $t^{\prime}, \bar{t}$, and $t$ are in cyclic order on the contour, i.e., they are ordered according to $t^{\prime} \prec \bar{t} \prec t$. Here, $\prec$ denotes the contour ordering indicated by the arrows in Fig. 2(b). For more details, see Ref. [49]. Thus, the impurity KBE becomes

$\left(i \partial_{t}+\lambda-E_{\alpha}\right) \mathcal{G}_{\alpha}\left(t, t^{\prime}\right)-\int_{\mathcal{C}, \text { cycl. }} d \bar{t} \Sigma_{\alpha}(t, \bar{t}) \mathcal{G}_{\alpha}\left(\bar{t}, t^{\prime}\right)=\delta_{\mathcal{C}}\left(t, t^{\prime}\right)$.

Here, $\lambda$ denotes the pseudoparticle chemical potential. Apart from the difference in the convolution integral, the lattice and impurity KBEs, Eqs. (4) and (6), respectively, have the same mathematical structure, and the same scaling of the computational effort with the number of time steps. In the following, we will discuss a generic truncation scheme that applies to both cases.

\section{B. Solution scheme and memory cutoff}

In this section, we briefly discuss the standard procedure for solving a generic KBE. The goal is to introduce a consistent way of truncating the memory.

Suppose we are dealing with the generic KBE along with its adjoint version

$$
\begin{aligned}
\left(i \partial_{t}-\varepsilon\right) G\left(t, t^{\prime}\right)-I\left(t, t^{\prime}\right) & =\delta_{\mathcal{C}}\left(t, t^{\prime}\right), \\
\left(-i \partial_{t}-\varepsilon\right) G\left(t^{\prime}, t\right)-\bar{I}\left(t^{\prime}, t\right) & =\delta_{\mathcal{C}}\left(t, t^{\prime}\right),
\end{aligned}
$$

where $I\left(t, t^{\prime}\right)\left[\bar{I}\left(t, t^{\prime}\right)\right]$ represents a convolution integral of a generic self-energy $\Sigma\left(t, t^{\prime}\right)$ with $G\left(t, t^{\prime}\right)\left[G\left(t, t^{\prime}\right)\right.$ with $\left.\Sigma\left(t, t^{\prime}\right)\right]$ as in Eq. (4) or (6). The first step in solving Eq. (7) is to project the contour time arguments onto real $\left(t, t^{\prime} \in \mathcal{C}_{ \pm}\right)$and imaginary $\left(t=-i \tau \in \mathcal{C}_{\mathrm{im}}\right)$ arguments. The different combinations give rise to the different Keldysh components of the GF. Our solution scheme is based on the retarded $\left[G^{\mathrm{R}}\left(t, t^{\prime}\right)\right]$, lesser $\left[G^{<}\left(t, t^{\prime}\right)\right]$, left-mixing $\left[G^{\rceil}(t, \tau)\right]$, and Matsubara $\left[G^{\mathrm{M}}(\tau)\right]$ components. All other Keldysh components can be obtained as linear combinations of the above. Convolutions can be expressed in terms of the Keldysh components by using the standard Langreth rules [25] for lattice-type KBEs or the modified Langreth rules for pseudoparticle GFs [49].

Assuming that the equilibrium problem has been solved and the Matsubara component $G^{\mathrm{M}}(\tau)$ is known, the time propagation of the real-time and left-mixing components can be performed according to the sketch in Fig. 3. We assume an equidistant discretization $t_{n}=n \Delta t$ of the real-time axis into $N_{t}$ steps of size $\Delta t$.

To compute the retarded component $G^{\mathrm{R}}\left(t_{n}, t_{j}\right)$ for all $j=$ $0, \ldots, n$ one can start from the diagonal $G^{\mathrm{R}}\left(t_{n}, t_{n}\right)$ which is known from the commutation relations of the creation and annihilation operators. Using the adjoint KBE (7b) and the Langreth rules, the retarded GF is propagated by

$$
-i \partial_{t} G^{\mathrm{R}}\left(t_{n}, t\right)=G^{\mathrm{R}}\left(t_{n}, t\right) \varepsilon+\int_{t}^{t_{n}} d \bar{t} G^{\mathrm{R}}\left(t_{n}, \bar{t}\right) \Sigma^{\mathrm{R}}(\bar{t}, t) .
$$

Hence, to obtain $G^{\mathrm{R}}\left(t_{n}, t_{j}\right)$, the retarded self-energy $\Sigma^{\mathrm{R}}\left(t_{i}, t_{j}\right)$ is needed for $i=j, \ldots, n$, i.e., the lattice points marked by the blue shaded background in Fig. 3(a). The self-energy $\Sigma^{\mathrm{R}}\left(t_{n}, t_{i}\right)$ for $i=0, \ldots, n$ is further needed to obtain the left-mixing component $G^{\uparrow}\left(t_{n}, \tau\right)$ and the lesser component $G^{<}\left(t_{j}, t_{n}\right)$. The propagation scheme for the latter [green arrows in Fig. 3(a)], starting from $G^{<}\left(0, t_{n}\right)$ and progressing towards the diagonal $G^{<}\left(t_{n}, t_{n}\right)$, additionally requires the lesser self-energy $\Sigma^{<}\left(t_{j}, t_{i}\right)$ for $i=0, \ldots, n$ for the calculation of $G^{<}\left(t_{j}, t_{n}\right)$ [lattice points marked by the green shaded background in Fig. 3(a)].

We note that the KBEs can also be solved with different propagation schemes, as explained, for instance, in Refs. [55-57]. The dependence of the memory on the selfenergy is, however, the same.

The computational effort for the solution of the KBEs (7) scales like $O\left(N_{t}^{3}\right)$ assuming that the self-energy is known. This is the bottleneck in calculations based on simple impurity 


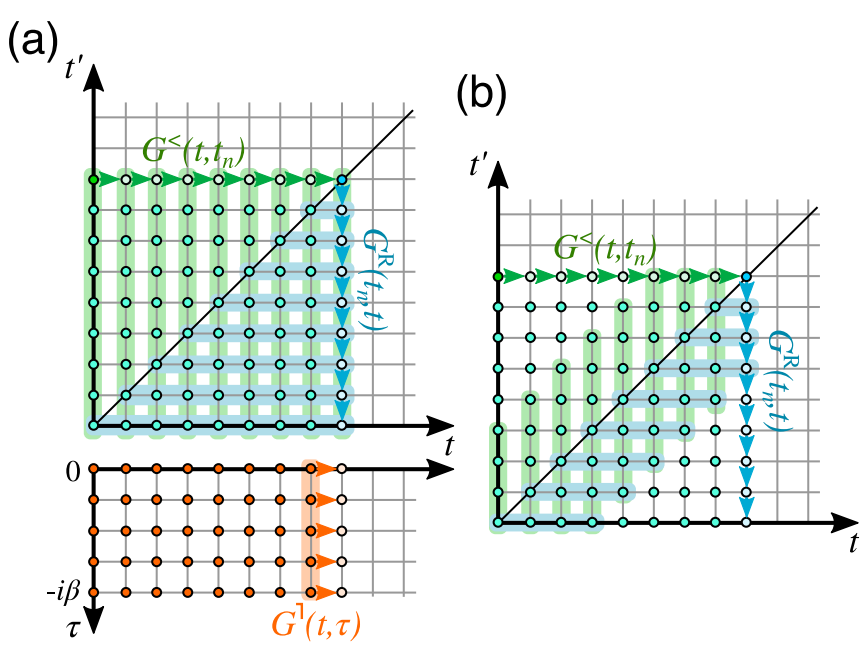

FIG. 3. (a) Sketch of the propagation scheme of the KBEs (7) for the retarded and lesser GFs in the two-time plane (upper part) and of the left-mixing GF (lower part). The method starts with the known value of $G^{\mathrm{R}}(t, t)$ at the diagonal, while successive time stepping (blue arrows) yields $G^{\mathrm{R}}\left(t, t^{\prime}\right)$ for all $t^{\prime}$ up to $t$. Once $G^{\mathrm{R}}(t, 0)$ is known, the KBE for $G^{\dagger}(t, \tau)$ can be solved. Finally, using $G^{<}(0, t)=$ $-\left[G^{\dagger}(t, 0)\right]^{\dagger}$, the lesser GF can be propagated up to $G^{<}(t, t)$. The shaded lattice points indicate the values of the self-energy which are needed to calculate one time step. (b) Simplified propagation scheme [analogous to (a)] for times $t$ larger than the cutoff $t_{\text {cut }}$.

solvers, such as the NCA. At the OCA level, the evaluation of the pseudoparticle self-energy involves two internal integrals over the contour $\mathcal{C}$, so that in this case the calculation of $\Sigma_{\alpha}$ at a cost $O\left(N_{t}^{4}\right)$ dominates simulation. In either case, the computational scaling can be reduced by at least one order if the memory time of the self-energy is truncated to $\left|t-t^{\prime}\right| \leqslant t_{\text {cut }}$. Physically, a truncation of the memory occurs, e.g., when the system is coupled to a bath without particle exchange. For instance, a bath with broad spectrum would lead to a long-time decay of the form $G^{\mathrm{R}}\left(t, t^{\prime}\right) \sim e^{-\eta\left(t-t^{\prime}\right)}, G^{<}\left(t, t^{\prime}\right) \sim$ $e^{-\eta\left|t-t^{\prime}\right|}$, and $G^{\rceil}(t, \tau) \sim e^{-\eta t}$, and analogously for the selfenergy. A consistent way to introduce a numerical memory cutoff is to mimic this behavior by replacing $\Sigma^{\mathrm{R}}\left(t, t^{\prime}\right) \rightarrow f(t-$ $\left.t^{\prime}\right) \Sigma^{\mathrm{R}}\left(t, t^{\prime}\right), \Sigma^{<}\left(t, t^{\prime}\right) \rightarrow f\left(\left|t-t^{\prime}\right|\right) \Sigma^{<}\left(t, t^{\prime}\right)$, and $\Sigma^{\top}(t, \tau) \rightarrow$ $f(t) \Sigma^{\rceil}(t, \tau)$, with a generic cutoff function $f(t)$. Here, we choose a Fermi-function-like cutoff

$$
f(t)=\frac{1}{1+\exp \left[\left(t-t_{0}\right) / T_{c}\right]},
$$

which interpolates between a hard cutoff $\left(T_{c} \rightarrow 0\right)$ and an exponential decay $\left(T_{c} \rightarrow \infty\right)$. The cutoff time $t_{\text {cut }}$ after which memory effects can be neglected is thus defined by $f\left(t_{\text {cut }}\right)$ falling below a specified threshold. Other choices of the cutoff function are, of course, possible. For instance, a Gaussian cutoff could also be employed. However, for an accurate cutoff scheme, we require (aside from a rapid decay for $t>t_{\text {cut }}$ ) that the short-time dependence is unaffected. For this reason, a cutoff function which is approximately constant at short times, as our choice Eq. (9), is preferable.

For $t_{n}>t_{\text {cut }}$, the two-time propagation scheme of the KBEs (7) simplifies significantly [Fig. 3(b)]. First, the left-mixing component $\Sigma^{\rceil}\left(t_{n}, \tau\right)$ can be omitted. For this reason, solving for $G^{\dagger}\left(t_{n}, \tau\right)$ is not required anymore. Furthermore, the calculation of the real-time Keldysh components requires only the information of the retarded and lesser self-energy [blue or green shaded background in Fig. 3(b), respectively] on a reduced time interval of length $t_{\text {cut }}$.

\section{Memory cutoff via hybridization function}

We stress that the cutoff scheme introduced in Sec. IIB is general and applies to different types of NEGF setups. To illustrate the effect, we focus in the following on DMFT calculations in the strong-coupling limit and regard the GFs and the self-energy in Sec. IIB as pseudoparticle quantities. For simplicity, we will consider an infinite-dimensional Bethe lattice and employ an NCA impurity solver. In this case, the DMFT self-consistency condition for bandwidth $4 v$ simplifies to $\Lambda_{\sigma}\left(t, t^{\prime}\right)=v^{2} G_{\text {loc }, \sigma}\left(t, t^{\prime}\right)$ [34], so that an explicit solution of the lattice KBE (4) is not needed. In this case, the cutoff is most conveniently introduced on the level of the hybridization function:

$$
\begin{aligned}
\Lambda_{\sigma}^{\mathrm{R}}\left(t, t^{\prime}\right) & \rightarrow f\left(t-t^{\prime}\right) \Lambda_{\sigma}^{\mathrm{R}}\left(t, t^{\prime}\right), \\
\Lambda_{\sigma}^{<}\left(t, t^{\prime}\right) & \rightarrow f\left(\left|t-t^{\prime}\right|\right) \Lambda_{\sigma}^{<}\left(t, t^{\prime}\right), \\
\Lambda_{\sigma}^{\top}(t, \tau) & \rightarrow f(t) \Lambda_{\sigma}^{\top}(t, \tau) .
\end{aligned}
$$

Since each pseudoparticle self-energy $\Sigma_{\alpha}\left(t, t^{\prime}\right)$ contains a factor $\Lambda_{\sigma}\left(t, t^{\prime}\right)$ or $\Lambda_{\sigma}\left(t^{\prime}, t\right)$, this implies a corresponding truncation of the pseudoparticle self-energies. We remark that reducing the two-time dependence of the hybridization function would also yield a significant reduction of the computational cost of evaluating the internal integrals for the OCA or higher approximations. The effort would then grow only like a power of the cutoff time $t_{\text {cut }}$, rather than a power of the maximum simulation time.

\section{RESULTS}

\section{A. Hubbard model: Paramagnetic phase}

We first consider the simple case of a Hubbard model in the paramagnetic phase. Nonequilibrium DMFT studies of this model have provided fundamental insights into the nonequilibrium properties of strongly correlated electron systems, including dynamical phase transitions [10], dielectric breakdown [58], impact ionization [41], and thermalization [40]. Here, we drive the system out of equilibrium by an interaction modulation of the form

$$
U(t)=U_{0}+\Delta U \sin \left(\omega_{0} t\right) f_{\mathrm{p}}(t),
$$

with amplitude $\Delta U$ and pulse envelope $f_{\mathrm{p}}(t)=\sin ^{2}\left(\omega_{0} t / 2 N_{c}\right)$ for $0<t<2 \pi N_{c} / \omega_{0}$. This form represents an $N_{c}$-cycle pulse. Similar excitations by modulating the Hubbard repulsion have been realized experimentally [59] and studied theoretically $[49,60,61]$. Here, we focus on quasiresonant excitations using a pulse with $N_{c}=10$ cycles and $\omega_{0}=U_{0}+2$, corresponding to transitions to the higher-energy part of the upper Hubbard band. In the small-gap regime, this can lead to the production of additional doublon-holon pairs by impact ionization [41]. In the Mott insulating Hubbard model these excitation processes are of generic character and can also be realized by driving the 
system with time-dependent electric fields [62] or a periodic modulation of the hopping (for instance via coherent phonon excitations $[63,64])$. The specific form of the excitation plays, however, only a minor role for the following discussion.

To quantify the effect of the memory cutoff (9) we calculate the time evolution (i) without imposing additional approximations, which yields the reference DMFT GF $G\left(t, t^{\prime}\right)$, and (ii) using the cutoff scheme (10) for the hybridization function, which yields the approximate GF $G_{\text {cut }}\left(t, t^{\prime}\right)$. The inverse temperature is set to $\beta=10$. In order to assess the effect of the truncation of the hybridization function, we calculate the norm of the difference $\left\|G-G_{\text {cut }}\right\|$ for different cutoff parameters $t_{0}, T_{c}$ and a maximum simulation time $t_{\max }=100$. One can define the norm of a two-time GF in different ways. We consider the difference on the last time slice according to the formula

$$
\begin{aligned}
\|G\|= & \frac{1}{t_{\max }} \int_{0}^{t_{\max }} d t\left|G^{<}\left(t_{\max }, t\right)\right| \\
& +\frac{1}{t_{\max }} \int_{0}^{t_{\max }} d t\left|G^{\mathrm{R}}\left(t_{\max }, t\right)\right| \\
& +\frac{1}{\beta} \int_{0}^{\beta} d \tau\left|G^{\dagger}\left(t_{\max }, \tau\right)\right| .
\end{aligned}
$$

Since the GF at the last time step depends on all the previous steps, the definition (12) provides a convenient way of comparing full two-time GFs. We have also tested the norm

$$
\begin{aligned}
\|G\|= & \frac{1}{t_{\max }^{2}} \int_{0}^{t_{\max }} d t \int_{0}^{t_{\max }} d t^{\prime}\left|G^{<}\left(t, t^{\prime}\right)\right| \\
& +\frac{1}{t_{\max }^{2}} \int_{0}^{t_{\max }} d t \int_{0}^{t} d t^{\prime}\left|G^{\mathrm{R}}\left(t, t^{\prime}\right)\right| \\
& +\frac{1}{t_{\max } \beta} \int_{0}^{t_{\max }} d t \int_{0}^{\beta} d \tau\left|G^{\urcorner}(t, \tau)\right|
\end{aligned}
$$

and found the results to be qualitatively similar to those obtained by Eq. (12). Since Eq. (12) is faster to evaluate, we employ it in the following analysis. Note that the norm difference $\left\|G-G_{\text {cut }}\right\|$ measures the absolute error of $G_{\text {cut }}$ with respect to the reference solution $G$. The magnitude of the error does not depend on wether the system is in equilibrium or excited, but on the accuracy only.

Before we study the dynamics in detail, let us consider the time dependence of the equilibrium hybridization function $\Lambda_{\sigma}^{\mathrm{R}}(t, 0) \equiv \Lambda^{\mathrm{R}}(t, 0)$, shown in Fig. 4 along with a bounding envelope function defined by $\left|\Lambda_{\sigma}^{\mathrm{R}}(t, 0)\right| \leqslant\left|\Lambda_{\sigma}^{>}(t, 0)\right|+\left|\Lambda_{\sigma}^{<}(t, 0)\right|$ $\left[=2\left|\Lambda_{\sigma}^{<}(t, 0)\right|\right.$ in the particle-hole symmetric case]. As one infers from the figure, the retarded hybridization function (and thus all other real-time components) decays rapidly. In the presence of a Mott gap we can decompose $\left|\Lambda_{\sigma}^{\mathrm{R}}(t, 0)\right|$ into a lower [corresponding to $\Lambda_{\sigma}^{<}(t, 0)$ ] and upper Hubbard band [corresponding to $\Lambda_{\sigma}^{>}(t, 0)$ ]. Both the lesser and greater components are defined by a Fourier transform over a frequency domain with semi-infinite support (in the limit of $\beta \rightarrow \infty$ ). The Paley-Wiener theorem [65] then implies that the decay of the hybridization function in real time is of the form $\Lambda_{\sigma}^{\gtrless}(t, 0) \sim$ $\exp \left(-B t^{\alpha}\right)$ with $0<\alpha<1$. Although the temperature is finite in our study, a subexponential decay of the envelope function is observed in Fig. 4 due to the finite gap size and the exponen-
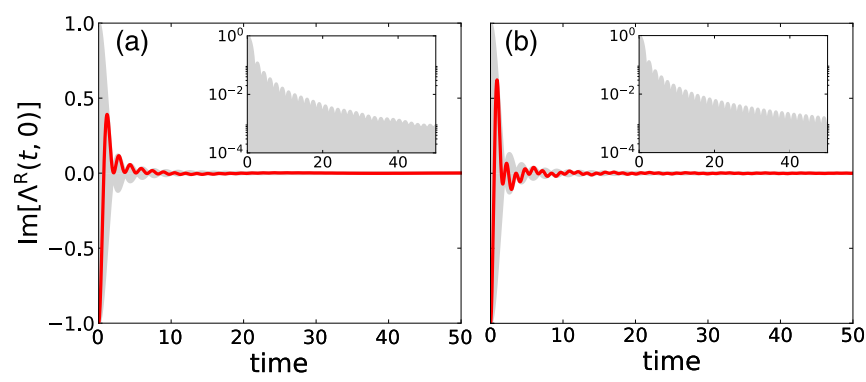

FIG. 4. Retarded component $\Lambda_{\sigma}^{\mathrm{R}}(t, 0)$ of the hybridization function in equilibrium for (a) $U_{0}=4$ and (b) $U_{0}=6$. The gray shaded background represents the envelope function $2\left|\Lambda_{\sigma}^{<}(t, 0)\right|$ (on a logarithmic scale in the inset).

tially vanishing spectral density within the gap. Nevertheless, since the pseudoparticle self-energy involves a multiplication with the (typically algebraically) decaying pseudoparticle GF, truncating the hybridization function at $t_{0} \sim 30$ is expected to yield a good approximation. In nonequilibrium calculations, the partial filling of the gap will result in a usual exponential decay, and the effective memory time will be further reduced.

As key quantities to assess the quality of the truncation approximation we consider, aside from the GF norm difference, also the (equilibrium or nonequilibrium) spectral functions obtained by the "backward" Fourier integration

$$
A(\omega, t)=-\frac{1}{\pi} \operatorname{Im} \int_{0}^{t} d t^{\prime} e^{i \omega\left(t-t^{\prime}\right)} G^{\mathrm{R}}\left(t, t^{\prime}\right),
$$

and, as an example of a local observable, the time-dependent double occupation $d(t)=\left\langle n_{\uparrow}(t) n_{\downarrow}(t)\right\rangle$. The results for $U_{0}=4$ (small-gap Mott insulator case) are shown in Fig. 5, while those for $U_{0}=6$ (larger-gap case) can be found in the Appendix (Fig. 14). Figures 5(a)-5(c) show the norm error in the Green's function for the equilibrium system $(\Delta U=0)$, a weak excitation pulse $\left(\Delta U=0.1 U_{0}\right)$ and a strong excitation pulse $\left(\Delta U=0.5 U_{0}\right)$, respectively. The color scale indicates the logarithm of the error, and the dashed contour lines correspond to fixed values of the error in the plane of $t_{0}$ and $1 / T_{c}$.

First of all, we notice that in this paramagnetic Hubbard model simulation, a sharp memory cutoff (large value of $1 / T_{c}$ ) has no particularly detrimental effect on the accuracy of the GF. Interestingly, however, the best approximations for the equilibrium system and the weakly excited system are obtained for a relatively well-defined cutoff temperature $T_{c}$ in the range $0.75-0.9$. As the excitation strength is increased, the optimal cutoff temperature becomes lower and less well defined.

The equilibrium system exhibits the largest cutoff effects. To reduce the norm error down to $10^{-3}$ one has to choose memory times $t_{0} \approx 30$, consistent with the rough estimate based on Fig. 4, and even this cutoff still produces small artifacts in the spectral function. This is illustrated in panel (d) of Figs. 5 and 14, which compare the exact spectral function (dashed) to the approximate spectral functions for the cutoff parameters indicated by the colored dots in Fig. 5(a). In particular, the sharp band edges are not well reproduced [see inset of Fig. 5(d)] and there appears some negative spectral weight in the gap region, which is unphysical. A hard cutoff leads to oscillations in the Fourier transformed self-energy. Via 
(a)
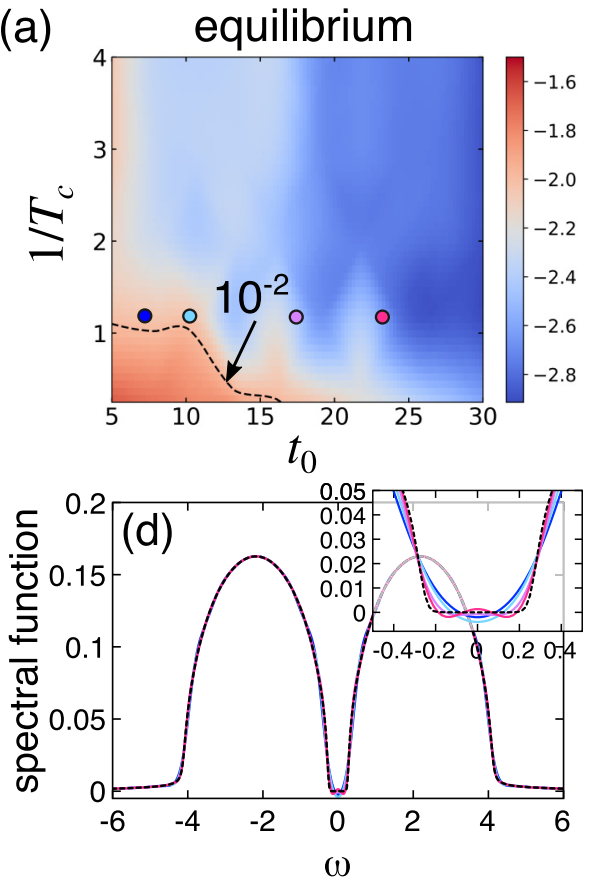

(b)
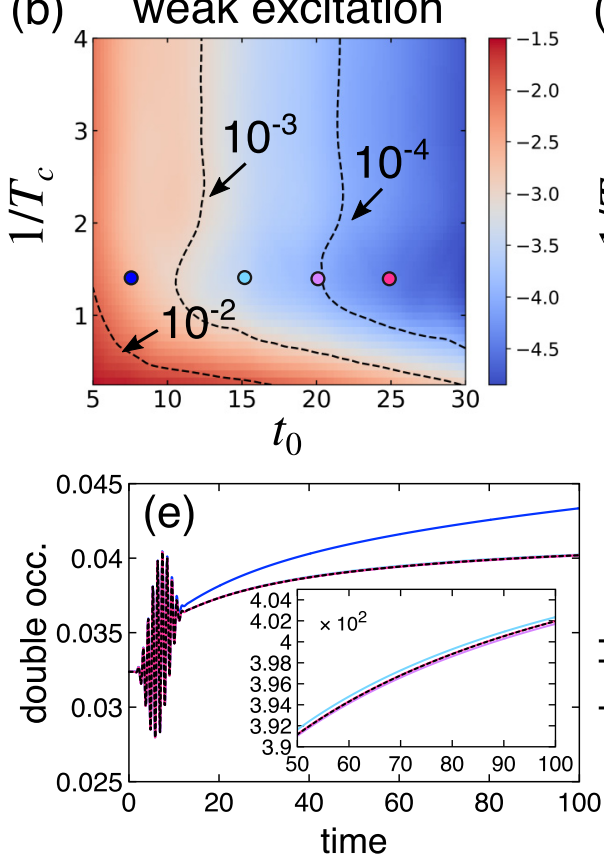
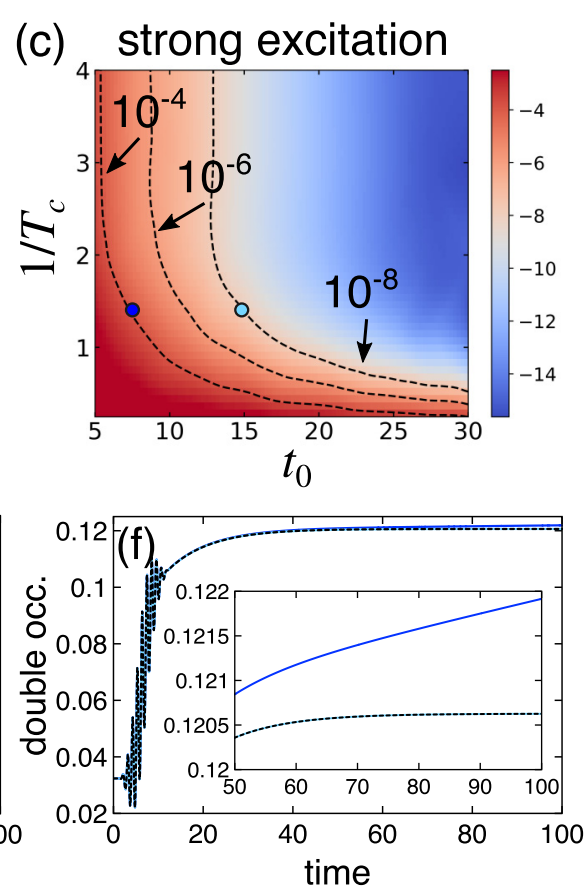

FIG. 5. (a)-(c) Norm distance (logarithm) between the two-time reference GF (no cutoff) and $G_{\text {cut }}$ obtained by the cutoff scheme as a function of the memory time $t_{0}$ and sharpness of the cutoff $1 / T_{c}$ [cf. (9)] for $U_{0}=4$ and $\Delta U=0$ (a), $\Delta U=0.4$ (b), and $\Delta U=2.0$ (c). The contour lines delimit the regions where the error is smaller than the given values. The colored dots indicate representative values of $t_{0}$ and $T_{c}$, for which the equilibrium spectral function (d) and the double occupancy for weak (e) and strong excitation (f) are shown (consistent color coding). The black dashed lines represent the reference results.

the Dyson equation, such features will also be visible in the spectral function. The approximate spectral function, however, approaches the exact result with increasing $t_{0}$, which shows that the truncation errors can be systematically controlled. Sharp spectral features are associated with slowly decaying Green's functions, and this in turn implies slowly decaying pseudoparticle Green's functions and self-energies. It is thus not surprising that the equilibrium system at low temperature represents a challenging test case for our cutoff scheme.

Simulations with truncated memory time are much more accurate in the nonequilibrium case. Even after a weak excitation [panel (b) in Figs. 5 and 14], errors in the GF of the order $10^{-4}$ can be reached with $t_{0}$ in the range 15-30 (depending on $U$ ) and this level of accuracy is sufficient to produce spectral functions and local observables that are, within the numerical accuracy of the simulation, hardly distinguishable from the results of the full calculations. In Fig. 5(e) we plot the time evolution of the double occupation, for the cutoff parameters indicated by the colored dots in Fig. 5(b). Already the light-blue curve $\left(t_{0}=15\right)$ is accurate to more than four digits (see inset), and this error remains constant up to the longest simulation times. Comparing the results of Figs. 5(e) and 14(e), we furthermore note that in the case of a small-gap insulator (Fig. 5) the thermalization dynamics involves two timescales. The fast timescale (i.e., the dynamics up to time $t \approx$ 40) can be associated with doublon-holon creation by impact ionization [41], while the longer (thermalization) timescale is associated with doublon-holon creation by multiparticle scattering. In the large-gap insulator (Fig. 14), the impact ionization is suppressed because the kinetic energy of the pulse-induced doublons and holons is not sufficient to produce additional doublon-holon pairs. The simulation with memory cutoff correctly reproduces this physics.

Finally, in the strong pulse excitation case, where a large amount of energy is injected into the system and the "photodoped" doublon density reaches several percent, the memory time becomes very short. In these simulations, $t_{0} \approx 10$ is sufficient to reproduce the exact results with 5 digits accuracy [see Figs. 5(c) and 5(f)].

The results for the nonequilibrium spectral function, plotted in Fig. 6, confirm the conclusions drawn from the previous analysis. In the weak excitation case [Figs. 6(a) and 6(c)] small artifacts appear in the gap region if the cutoff time is chosen too small $\left(t_{0}=7.5\right.$ ), while $t_{0}=15$ is sufficient to reproduce the exact time-dependent spectra. In the case of a strong excitation [Figs. 6(c) and 6(d)], even $t_{0}=7.5$ is adequate.

In general, stronger excitations result in a partial filling of the Mott gap as a result of heating and photodoping. For this reason, the Paley-Wiener theorem does not apply any more, and the (nonequilibrium) hybridization functions $\Lambda_{\sigma}^{\gtrless}\left(t, t^{\prime}\right)$ decay exponentially in $\left|t-t^{\prime}\right|$. Hence, truncating the memory works better for stronger excitations.

\section{B. Hubbard model: Antiferromagnetic phase}

As the next paradigmatic example we study the Hubbard model in the AFM phase, which can be stabilized by the selfconsistency condition $\Lambda_{\sigma}\left(t, t^{\prime}\right)=v^{2} G_{\mathrm{loc}, \bar{\sigma}}\left(t, t^{\prime}\right)$. We choose the same excitations as in Sec. III A and consider the equilibrium system, and the case of weak and strong perturbation. Here, a smaller time step of $\Delta t=0.01$ is required for a stable 

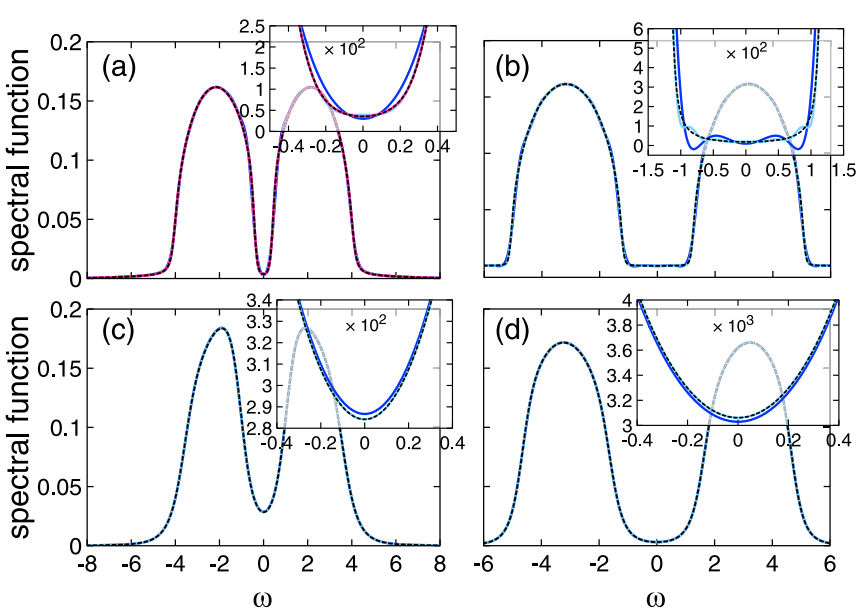

FIG. 6. Nonequilibrium spectral functions computed at $t_{\max }=$ 100 for (a) $U_{0}=4, \Delta U=0.4$, (b) $U_{0}=6, \Delta U=0.6$, (c) $U=4$, $\Delta U=2.0$, and (d) $U=6, \Delta U=3$. Different lines correspond to different cutoff parameters $t_{0}$ with fixed $T_{c}$. The color coding corresponds to the circle symbols shown in Fig. 5(b) [for panels (a) and (b)] and Fig. 5(c) [for panels (c) and(d)]. The insets show a zoom of the gap region. For the case of strong excitation [(c) and (d), respectively], the spectral functions obtained by the cutoff scheme are identical to the reference one up to $10^{-4}$.

propagation, hence, we compute the DMFT solution up to $t_{\text {max }}=50$.

We start again by analyzing the retarded component of the equilibrium hybridization function, which yields the spectral function by Fourier transformation. The result, shown in Fig. 7 , is quite different from the paramagnetic case. Long-lived oscillations are present, which give rise to characteristic sharp spectral features associated with antiferromagnetic excitations. Inspecting the decay of the envelope function for $U_{0}=4$ [Fig. 7(a)], which stays above $10^{-2}$ up to $t_{\max }$, it appears that any truncation of the memory should result in a quite poor approximation. This "worst case" scenario allows us to investigate the artifacts introduced by truncating the memory at too early times. It turns out that a sufficiently large cutoff time $<t_{\max }$ nevertheless captures the main features and yields a good approximation of the equilibrium properties and, in particular, the nonequilibrium dynamics. The quality of the cutoff approximation can be expected to substantially improve

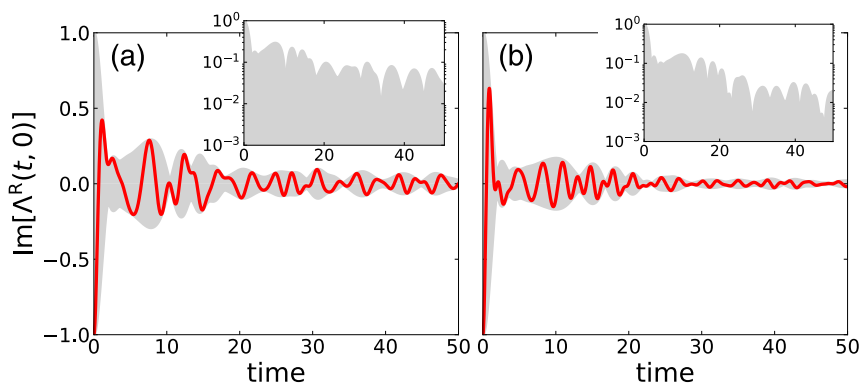

FIG. 7. Retarded component $\Lambda_{\uparrow}^{\mathrm{R}}(t, 0)$ of the hybridization function in equilibrium (AFM case) for (a) $U_{0}=4$ and (b) $U_{0}=6$. The gray shaded background represents the envelope function $\left|\Lambda_{\uparrow}^{>}(t, 0)\right|+$ $\left|\Lambda_{\uparrow}^{<}(t, 0)\right|$ (on a logarithmic scale in the inset). for $U_{0}=6$ [Fig. 7(b)] since in this case the hybridization function approaches zero faster.

Figure 8 presents an analysis of the small-gap system analogous to Sec. III A, while the results for larger gap can be found in the Appendix (Fig. 15). In the equilibrium case, as expected from the slow decay of the hybridization function, the norm error of the GF produced by the cutoff scheme is generally larger than for the paramagnetic case [Figs. 8(a)-8(c)]. In particular, the error does not fall below $10^{-2}$. Furthermore, the time propagation for $\Delta t=0.01$ can become unstable for certain cutoff parameters ( $t_{0}$ and $T_{c}$ chosen in the black regions of the figure). Inspecting the time-dependent observables, which should be constant in equilibrium, we found that the total density $\left\langle\hat{n}_{\uparrow}\right\rangle+\left\langle\hat{n}_{\downarrow}\right\rangle$ is conserved up to an accuracy of $\sim 10^{-5}$ or better for any values of the cutoff parameters. In contrast, the AFM order parameter $\left\langle\hat{n}_{\uparrow}\right\rangle-\left\langle\hat{n}_{\downarrow}\right\rangle$ violates the corresponding conservation law (depending on the cutoff parameters). In particular, for too small values of $t_{0}$, the magnitude of the AFM order decreases. This effect is most pronounced in the unstable region. The instability is related to negative spectral weight originating from a sharp cutoff $\left(T_{c}>1.5\right)$. In fact, multiplying the exact reference hybridization function with the cutoff according to Eq. (10) and performing the Fourier transformation, one obtains a spectral function with negative weight in the gap region. This breaks the conservation of $\left\langle\hat{n}_{\sigma}\right\rangle$ and therefore of the order parameter.

Inspecting the equilibrium spectral functions [Fig. 8(d)] obtained by the time evolution with memory cutoff, we find sharp spectral features originating from antiferromagnetic excitations, which are the reason for the slow decay of $\Delta_{\sigma}\left(t, t^{\prime}\right)$ and the difficulties of the cutoff procedure in the equilibrium case. In particular, negative spectral weight appears in the gap for $T_{c}=0.83$ and $t_{0}=12$ (blue line). Increasing $t_{0}$, the cutoff results still deviate substantially from the exact spectrum, unless the cutoff time $t_{0}$ is increased up to $t_{0} \approx 27$.

The situation improves after a weak excitation [Fig. 8(b)]. Apart from an unstable region at small $t_{0}$, the error can be reduced to less than $10^{-3}$ for cutoff times $t_{0}>20$ for $1 / T_{c} \approx$ 0.82 , while further increasing $t_{0}$ yields even smaller errors. As a relevant local observable, we compare in Fig. 8(e) the AFM order parameter obtained for different cutoffs. We find that $t_{0} \approx$ 20 is sufficient to converge the dynamics of the order parameter to an absolute deviation of less than $10^{-3}$, even though in this excitation regime the order parameter is reduced by only about 20\%. After a strong excitation [Fig. 8(c)], on the other hand, the magnetic order melts rapidly, such that the behavior discussed for the paramagnetic system in Sec. III A is recovered to a large extent. The evolution of the order parameter is shown in Fig. 8(f) and deviates by less than $10^{-5}$ from the exact solution.

Increasing the Hubbard repulsion to $U_{0}=6$ (see Appendix, Fig. 15), one finds a similar behavior as for $U_{0}=4$ except that the artifacts in the equilibrium case [Figs. 15(a) and 15(d)] are less pronounced. The equilibrium time propagation with time step $\Delta t=0.01$ is stable for $t_{0}>12$ for all $T_{c}$ and the error is reduced below $10^{-2}$ for $t_{0}>25$ and small enough $T_{c}$. Inspecting the equilibrium spectral functions, good agreement is found for $t_{0}>20$. This can be understood from the faster oscillations of the hybridization function (corresponding to spectral features at larger $|\omega|$ ). If there are more oscillations in a given time interval, the negative spectral weight arising 

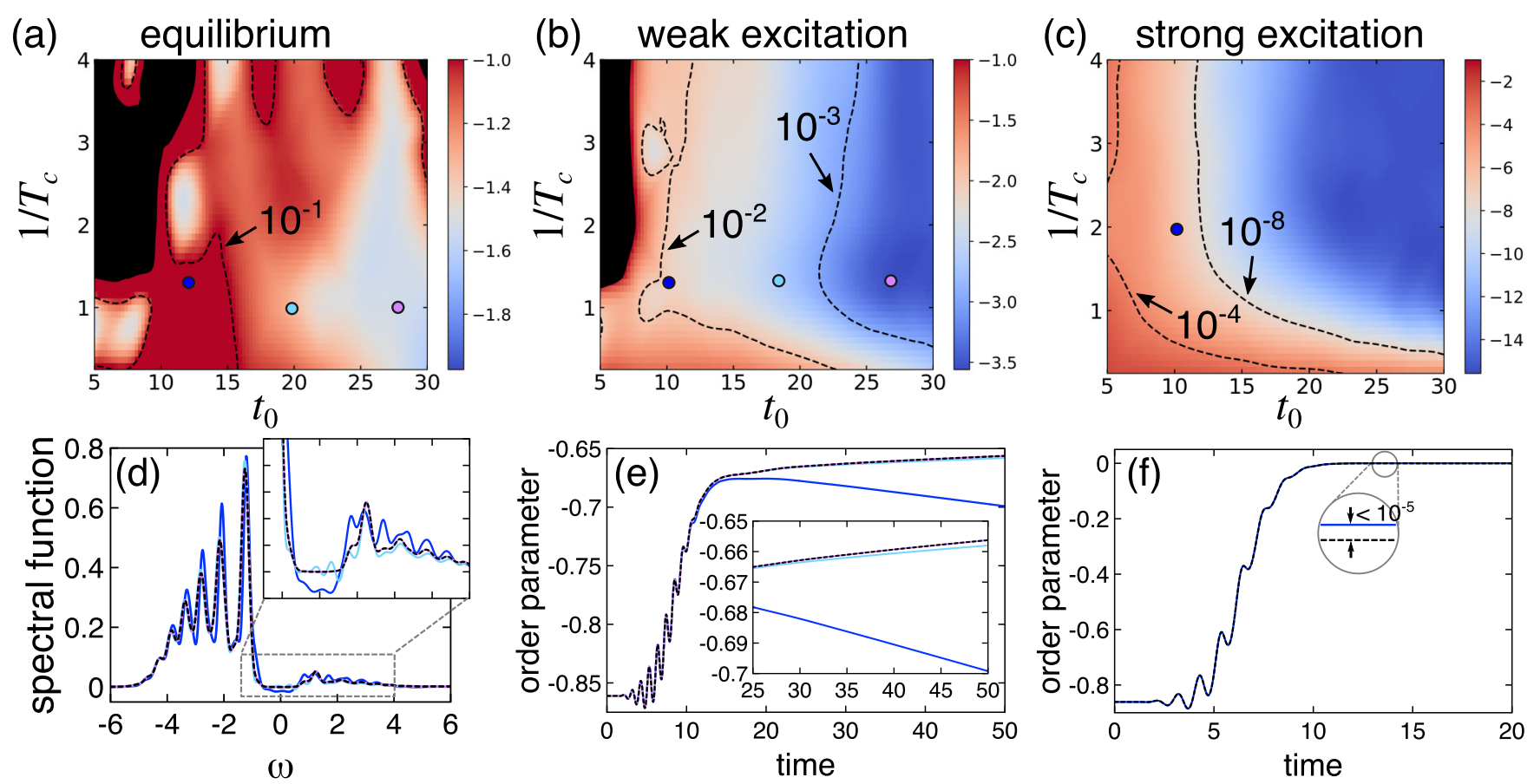

FIG. 8. (a)-(c) Norm distance (logarithm) between the two-time reference GF $G_{\text {loc, } \uparrow}\left(t, t^{\prime}\right)$ (no cutoff) and the GF obtained by the cutoff scheme, analogous to Fig. 5, for $U_{0}=4$ and $\Delta U=0$ (a), $\Delta U=0.4$ (b), and $\Delta U=2.0$ (c) in the AFM phase. The black region indicates cutoff parameters for which the propagation with time step $\Delta t=0.01$ is unstable. (d) Equilibrium majority-spin spectral function obtained from the reference GF (black dashed line) and the cutoff scheme [colors as in (a)]. The dynamics of the AFM order parameter $\left\langle\hat{n}_{\uparrow}\right\rangle-\left\langle\hat{n}_{\downarrow}\right\rangle$ is shown for $\Delta U=0.4$ in (e), and for $\Delta U=2.0$ in (f).

from a cutoff is reduced. Furthermore, the peaks of the spectral function are less pronounced than for $U_{0}=4$. The error of the GF for weak excitations [Fig. 15(b)] can be suppressed to less than $10^{-3}$ by choosing $t_{0}>15$. For $t_{0}>25$, the AFM order parameter deviates by less $\sim 10^{-4}$ from the exact result. Again, the behavior after a strong excitation [Figs. 15(c) and 15(f)] resembles the strongly excited paramagnetic case, where the memory of the hybridization functions drops rapidly and leads to excellent approximations even for short cutoff times.

We have also computed the nonequilibrium spectral function at $t=t_{\max }$ for the case of weak excitations (Fig. 9). Due to the reduction of the AFM order parameter, the sharp spectral features are washed out, and we find a good agreement with the reference spectral function for moderate values of $t_{0}$. Nevertheless, for an accurate estimate of the the electronic gap, $t_{0} \approx 18$ is required.

In order to study nonequilibrium dynamics specifically associated with symmetry-broken phases we perform a scan over $\Delta U$. Depending on the excitation strength one observes two qualitatively different behaviors of the AFM order parameter, and diverging timescales near the critical excitation amplitude. This is a manifestation of a dynamical phase transition. We note that the latter term has been used in various contexts $[10,66-68]$ and that numerical simulations have played an important role in revealing these phenomena. In this study, the nonequilibrium transition is between two long-lived nonthermal states with and without AFM order, and the (almost) diverging timescale is associated with the melting of the order, or the relaxation into the trapped AFM state [69]. The universal character of this type of dynamical phase transition is exemplified by its appearance in a broad range of systems, including superconductors [70-73], excitonic insulators [74], antiferromagnetic [14,69], ferromagnetic
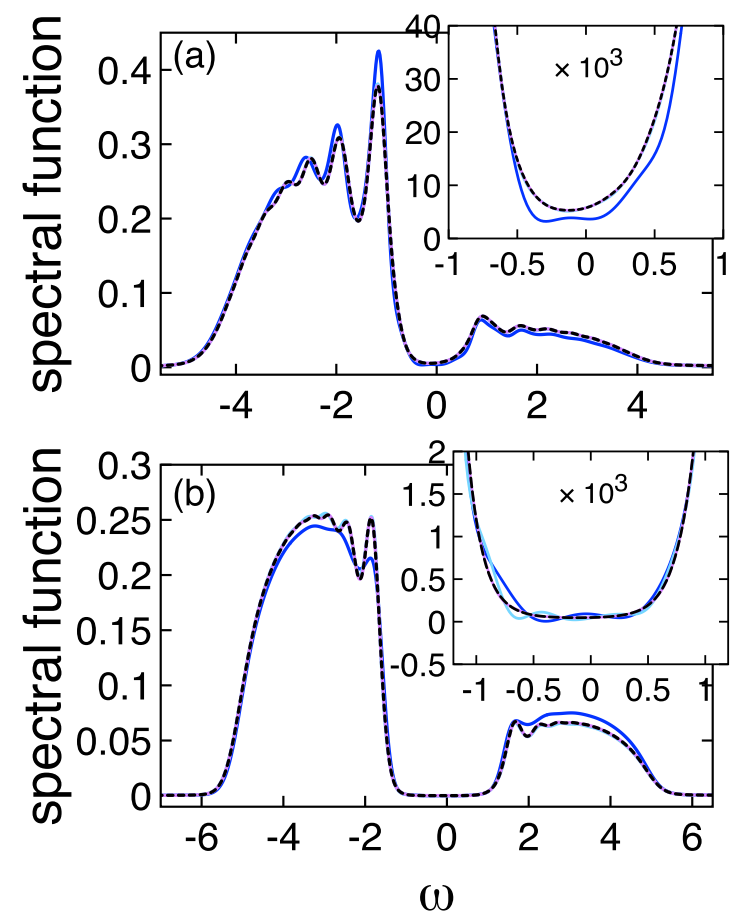

FIG. 9. Nonequilibrium (spin-up) spectral functions computed at $t_{\max }=50$ for (a) $U_{0}=4, \Delta U=0.4$ and (b) $U_{0}=6, \Delta U=0.6$. Different lines correspond to different cutoff parameters $t_{0}$ and $T_{c}$. The color coding corresponds to the circle symbols shown in Figs. 8(b) [for panel (a)] and 15(b) [for panel (b)]. 

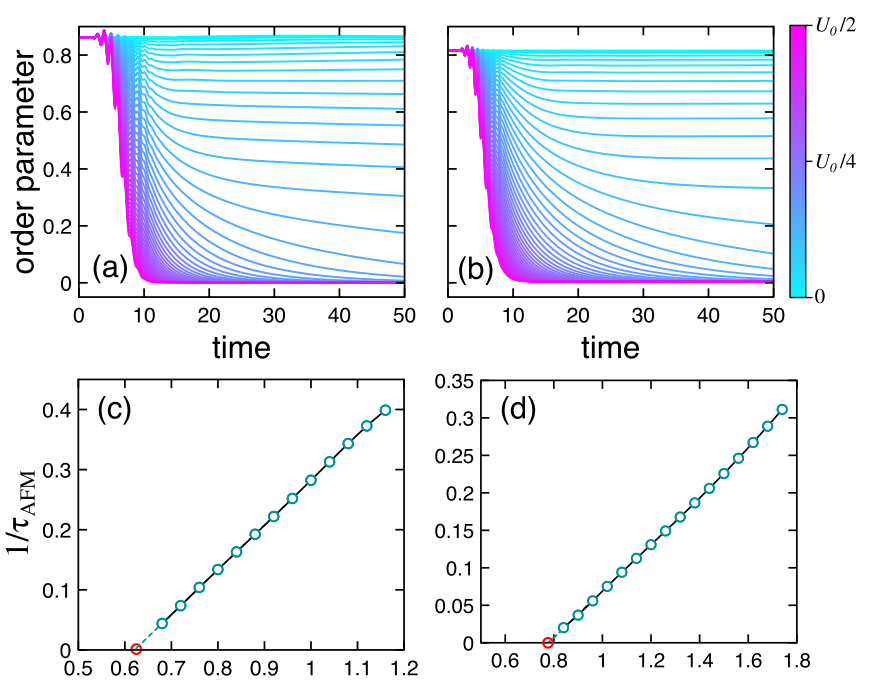

$\Delta U$

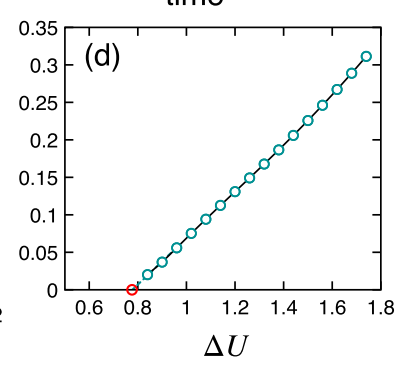

FIG. 10. Time-dependent AFM order parameter as a function of the excitation strength $\Delta U$ (color coding) for (a) $U_{0}=4$ and (b) $U_{0}=6$. The inverse decay rate $\tau$ of the AFM order parameter obtained with $t_{0}=15$ and $T_{c}=0.8$ (circles) is shown in (c) for $U_{0}=4$ and in (d) for $U_{0}=6$. The black solid line shows the results obtained without any cutoff, while the dashed line indicates the linear extrapolation to the critical point where $1 / \tau_{\mathrm{AFM}}=0$ (marked by the red circle).

[11,68,75], and charge-ordered [15] systems. Figure 10 shows the dynamics of the AFM order parameter as a function of $\Delta U$ for both the small-gap [Fig. 10(a)] and the wide-gap case [Fig. 10(b)] with cutoff parameters $t_{0}=15$ and $T_{c}=$ 0.8 . The behavior is qualitatively similar for both values of $U_{0}$ : For $\Delta U<0.1 U_{0}$, the order parameter exhibits amplitude oscillations after the pulse $(t \gtrsim 10)$ with a frequency related to the gap size. With increasing excitation strength, the oscillation frequency decreases and becomes difficult to measure near the nonthermal critical point. Testing different values of the cutoff parameters, we found that an accurate description of the regime of amplitude mode oscillations requires $t_{0} \approx 25$, especially for $U_{0}=4$. This is consistent with the error analysis in Fig. 8. In contrast, the regime of stronger excitations, above the excitation threshold for nonthermal melting of the AFM, is well captured by a memory time as short as $t_{0}=15$. As can be seen in Fig. 10, a strong excitation $\Delta U \approx 0.5 U_{0}$ results in a rapid melting of the AFM order. Decreasing $\Delta U$, one approaches the nonthermal critical point and the melting time increases. Note that the time dependence of the order is determined by two processes: nonthermal melting at shorter times followed by thermalization at longer times $(t \gtrsim 100)$. Here, we focus on the decay of the order at times after transient effects due to the pulse have vanished $(t>20)$, but before the thermalization stage $(t<50)$. An exponential fit allows us to extract the characteristic timescale $\tau_{\mathrm{AFM}}$, whose inverse $\tau_{\mathrm{AFM}}^{-1}$ is plotted in Figs. 10(c) and 10(d). One finds a linear behavior of $\tau_{\mathrm{AFM}}^{-1}$ as a function of $\Delta U$, in agreement with Ref. [69]. The extrapolation to zero defines the nonthermal critical point on the $\Delta U$ axis. We find, for both values of $U_{0}$, the critical amplitude $\Delta U \simeq 0.133 U_{0}$. Testing different cutoff parameters confirmed that $t_{0}=15$ is enough to determine $\tau_{\mathrm{AFM}}^{-1}$ within the numerical accuracy of the nonequilibrium DMFT simulation,

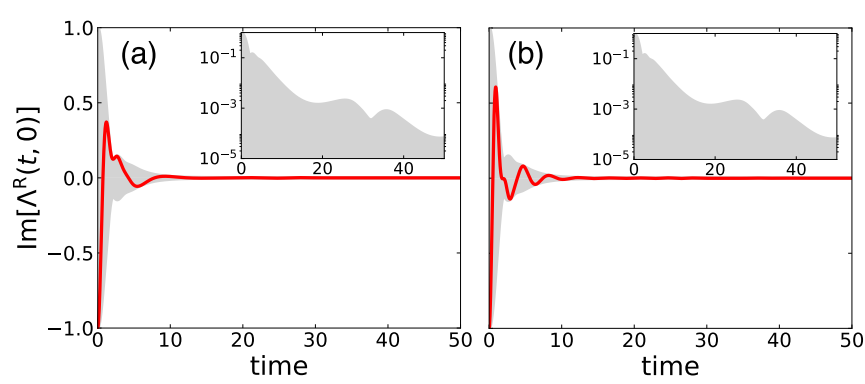

FIG. 11. Retarded component $\Lambda_{\sigma}^{\mathrm{R}}(t, 0)$ of the hybridization function in equilibrium for (a) $U_{0}=4$ and (b) $U_{0}=6$. The gray shaded background represents the envelope function $\left|\Lambda_{\sigma}^{>}(t, 0)\right|+\left|\Lambda_{\sigma}^{<}(t, 0)\right|$ (on a logarithmic scale in the inset).

as Figs. 10(c) and 10(d) demonstrate. This illustrates the potential of the cutoff scheme in studies of dynamical phase transitions and nonthermal dynamics.

\section{Holstein-Hubbard model}

We now proceed to the case of the Hubbard-Holstein model, which corresponds to the Hamiltonian (1) with $g>0$. The Hubbard-Holstein model can be treated within the framework of DMFT by applying the Lang-Firsov transformation [76], which (within NCA) maps the problem to an effective impurity problem similar to that of the Hubbard model, up to an additional boson Green's function which multiplies the hybridization function in the pseudoparticle Dyson equations. We have propagated the nonequilibrium DMFT scheme up to $t_{\max }=100$, using a discretization of $\Delta t=0.02$. As for the Hubbard model, we study a small-gap insulator $\left(U_{0}=4.0\right)$ and a large-gap system $\left(U_{0}=6.0\right)$. The phonon frequency is chosen as $\omega_{\mathrm{ph}}=0.2$, while the e-ph coupling is fixed to $g=0.2$. Hence, the Lang-Firsov parameter is $g / \omega_{\mathrm{ph}}=1$ while the effective phonon coupling is $\lambda=g^{2} / \omega_{\mathrm{ph}}<1$, justifying the applicability of the NCA treatment using the Lang-Firsov transformation.

Figure 11 depicts the retarded hybridization function along with its bounding function for the equilibrium case. One finds a rapid decay which is even faster than for the paramagnetic Hubbard model. This is understood by the additional broadening introduced by the e-ph coupling, albeit phonon-induced oscillations lead to a nonmonotonic behavior. Truncating the memory is hence expected to be a good approximation.

The results for $U_{0}=4$ are summarized in Fig. 12. First, one notices an unstable region for $1 / T_{c} \approx 2$ and $t_{0}<10$, in which the solution diverges. The origin of this instability is similar to the AFM case: a sharp cutoff $\left(T_{c}<0.5\right)$ of the oscillating hybridization function leads to negative spectral weight, which indicates a violation of particle number and spectral weight conservation. Apart from this potentially unstable region of small cutoff times, the error is of the order $10^{-3}$ for most values of $T_{c}$ and $t_{0}$, but decreases very slowly with $t_{0}$. This behavior is very similar for equilibrium [Fig. 12(a)], and weak [Fig. 12(b)] and strong excitation [Fig. 12(c)]. In contrast to the paramagnetic Hubbard model, the e-ph coupling leads to a rapid dissipation of kinetic energy, i.e., efficiently cooling the photoexcited doublons. For this reason, the filling of the gap is far less pronounced than for the pure Hubbard model, and the 


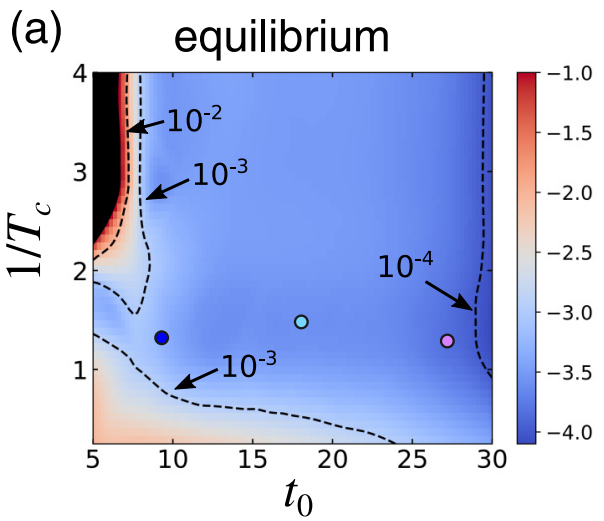

(b) weak excitation
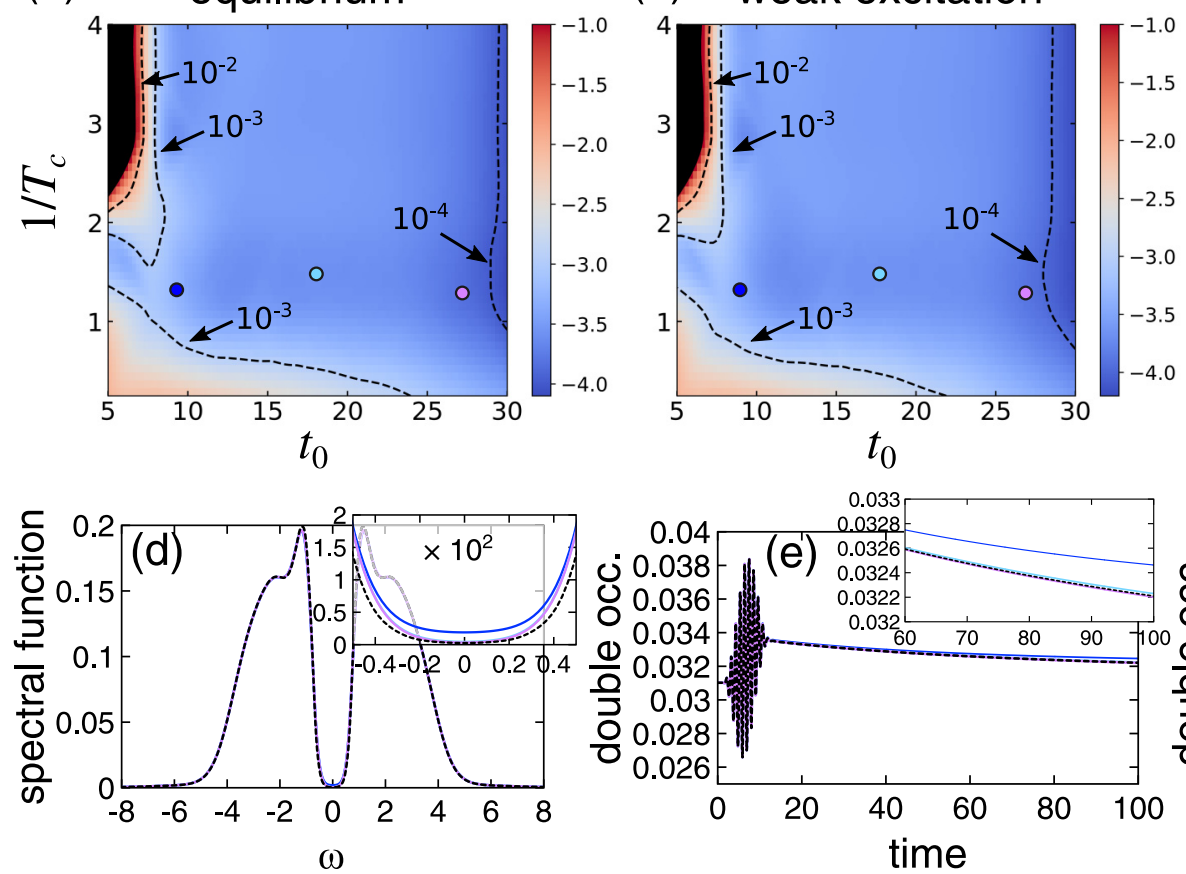

(c) strong excitation
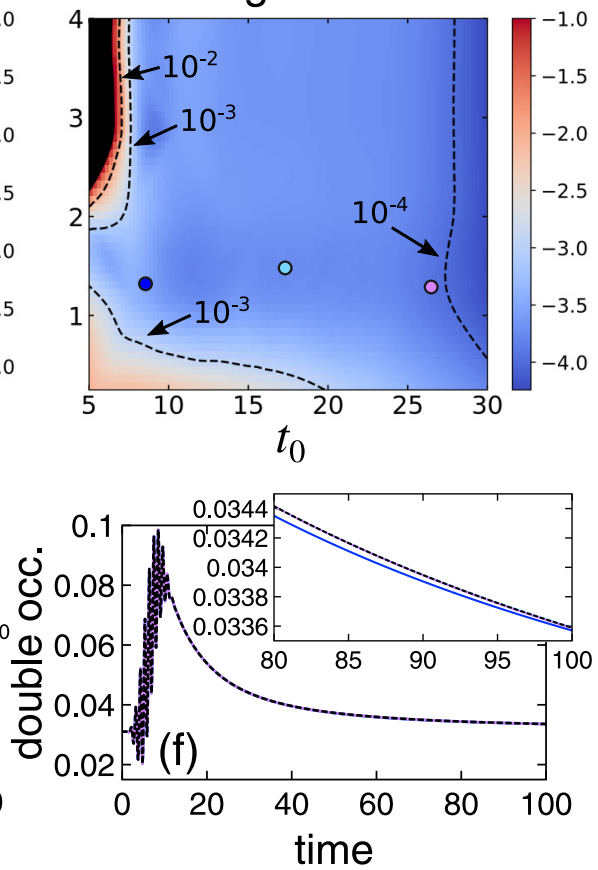

FIG. 12. (a)-(c) Norm distance (logarithm) between the two-time reference GF $G_{\text {loc, } \uparrow}\left(t, t^{\prime}\right)$ (no cutoff) and the GF obtained by the cutoff scheme for $U_{0}=4$ and $\Delta U=0$ (a), $\Delta U=0.4$ (b), and $\Delta U=2.0$ (c). (d) Equilibrium spin-up spectral function from the reference GF (black dashed) and the cutoff scheme [colors as in (a)]. The dynamics of the double occupation is shown in (e) for $\Delta U=0.4$ and (f) $\Delta U=2.0$.

spectral functions of the excited system are very similar to the equilibrium case.

Nevertheless, local observables such as the double occupation [Figs. 12(e)-12(f)] can be converged to match the exact propagation up $\sim 10^{-5}$ by increasing the cutoff time to $t_{0} \approx 17$. The cutoff temperature $1 / T_{c}$ plays only a minor role for large enough $t_{0}$, although there is an optimal region around $T_{c} \approx 0.6$, which provides a good compromise between not affecting the short-time dynamics and suppressing the hybridization function for larger $\left|t-t^{\prime}\right|$ in a smooth way.

We have also analyzed the case $U_{0}=6$ (see Fig. 16 in the Appendix), which is qualitatively similar to $U_{0}=4$. The error of the spectral functions and especially of the double occupation is, however, reduced by one order of magnitude. This is analogous to the observations for the Hubbard model (Fig. 14). Moreover, there is no unstable region in this case.

We have further analyzed the cooling dynamics induced by e-ph coupling and the ability of the cutoff scheme to reproduce the corresponding timescales. In these calculations, we use a larger Hubbard repulsion $U_{0}=10$ to exclude impact ionization processes and heating by doublon-holon recombination. Without e-ph coupling, the kinetic energy and the double occupation stay effectively constant. In contrast, including e-ph interactions, the photoexcited doublons can efficiently dissipate their kinetic energy $E_{\text {kin }}$ as long as it is larger than the phonon energy $\omega_{\mathrm{ph}}$. A further decrease of $E_{\text {kin }}$ becomes inefficient since no full quanta of phonon excitations can be emitted (so-called phonon bottleneck $[19,77])$. Therefore, two timescales for the relaxation of $E_{\text {kin }}$ are expected. Figure 13(a) shows the dynamics of the kinetic energy after a strong pulse excitation $\left(\Delta U=0.5 U_{0}\right)$ for $t_{0}=25$ and $T_{c}=0.75$. Comparing with the reference solution, one finds a difference of at most
$10^{-4}$. Analyzing the relaxation dynamics, a single-exponential decay is found for a small magnitude of the e-ph coupling $g$, while two different decay regimes become apparent for larger $g$. A double-exponential fit yields the decay constants $\gamma_{1}$ (fast relaxation) and $\gamma_{2}$ (slow relaxation), presented in Fig. 13(b). Consistent with Ref. [20], one finds a quadratic scaling of $\gamma_{1}$ with $g$, while the slow relaxation described by $\gamma_{2}$ does not increase significantly with $g$ for $g>0.3$. Comparing the decay constants for the smaller value of the memory time $t_{0}=15$ and the larger value $t_{0}=25$, small deviations are visible for larger $g$. Aside from these small differences, $\gamma_{1}$ and $\gamma_{2}$ are well reproduced even by $t_{0}=15$. Comparing with the reference solution we found the error of $\gamma_{1,2}$ to be less than $10^{-4}$ for $t_{0}=25$.
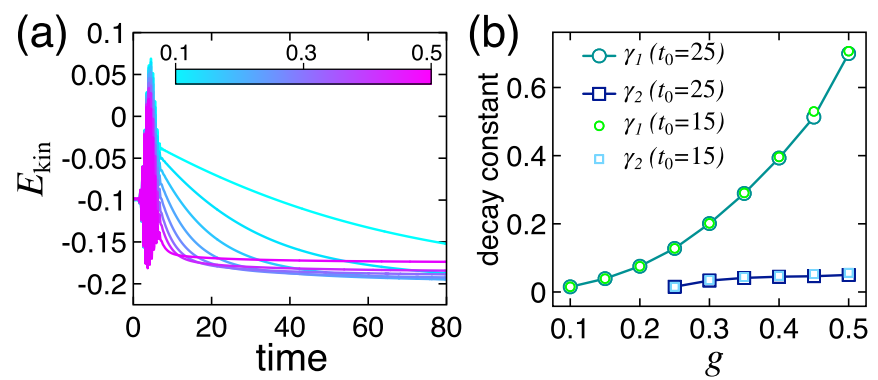

FIG. 13. (a) Dynamics of the kinetic energy for $U_{0}=10, \Delta U=$ $5, \omega_{\mathrm{ph}}=1$, and $g$ ranging from 0.1 to 0.5 (color scale). (b) The two exponential decay constants of the kinetic energy observed in (a), for cutoff time $t_{0}=25$ (large symbols and lines) and $t_{0}=15$ (smaller symbols). 


\section{CONCLUSIONS}

We studied the effect of truncations of the memory kernel in Kadanoff-Baym equations, with a focus on nonequilibrium DMFT simulations. These calculations are based on the solution of lattice and impurity Dyson equations in which a lattice, impurity, or pseudoparticle self-energy plays the role of a memory kernel. The standard techniques for the numerical solution of these equations involve a discretization of the time interval of length $t_{\max }$ into $N_{t}$ time slices of length $\Delta t$ and their computational cost (for given self-energy) scales as $N_{t}^{3}$. If the memory time of the self-energy is truncated at some $t_{\text {cut }}$ (corresponding to $N_{c}$ time slices), the computational effort can be reduced to $O\left(N_{c} N_{t}^{2}\right)$. A further reduction to $O\left(N_{c}^{2} N_{t}\right)$ is possible if the two-time GF is only calculated on $N_{c}$ time slices, e.g., because one is only interested in time-local observables. The appealing feature of the self-energy truncation approach is its simplicity, and that in addition to the speedup in the calculations, it also enables a potentially significant reduction in the memory requirements. An alternative route to overcome the memory bottleneck could be the use of a suitable compressed representation of the two-time self-energy [78], but such schemes are difficult to implement in a controlled way.

Considering typical parameter choices for nonequilibrium DMFT simulations of the Hubbard model in the strongly interacting regime, we have found that a memory time of less than 10 inverse hoppings is fully adequate to describe the time evolution of the system after a strong perturbation. In the case of weak perturbations, e.g., photodoping concentrations of less than 1\%, memory times of 20-30 inverse hoppings are needed to reduce the truncation error to a negligible level. (In practice, we found that a norm error of the Green's function $\lesssim 10^{-4}$ results in negligible effects on relevant observables.) As expected, the longest memory times are found in situations where the (nonequilibrium) spectral function exhibits sharp features, as is the case in the antiferromagnetic Mott state, or in equilibrium Mott insulators at low temperature, which feature sharp band edges. The coupling to phonons results in a slower convergence to the exact result with $t_{\text {cut }}$, especially in the strongly excited regime, because the system is cooled down by the phonons and the original gapped spectrum is recovered in the long-time limit. While a memory time of 10 yields a norm error in the Green's function of $10^{-3}, t_{\text {cut }} \approx 30$ is needed to reduce this error to $10^{-4}$.

While one might naively expect that smooth cutoffs of the memory time reduce artifacts, we found that the sharpness of the cutoff has little effect on the quality of the approximation in the paramagnetic case, even though in the equilibrium and weakly perturbed systems a nontrivial optimal "cutoff temperature" of the order of $T_{c} \approx 0.5-0.75$ could be identified. In the antiferromagnetic system, where the hybridization function decays more slowly, the sharpness of the cutoff matters, but for a large enough cutoff time, the same cutoff temperatures as in the paramagnetic Hubbard model are adequate.

We have demonstrated that simulations with memory cutoff correctly reproduce the different characteristic timescales appearing in the nonequilibrium evolution of photoexcited strongly correlated lattice systems. Not surprisingly, this is true for fast processes, such as the "photodoping" by the $U$ modulation, the generation of additional doublon-holon pairs by impact ionization, or the rapid cooling of the photocarriers in the presence of strong electron-phonon coupling. These occur on the timescale of a few inverse hoppings, which is comparable to the memory cutoff time. More remarkable is the fact that also the slower processes, in particular the thermalization timescale associated with doublon-holon production or recombination or the slow melting of AFM order near the nonthermal critical point, are accurately captured by the cutoff scheme. This suggests that under generic conditions, we can correctly reproduce the full nonequilibrium DMFT dynamics, including all the relevant fast and slow processes, as well as transient trapping phenomena, using a numerically efficient memory truncation scheme with $t_{\text {cut }} \ll t_{\text {max }}$.

The short memory times $t_{\text {cut }} \lesssim 10$ in strongly excited systems furthermore imply huge potential efficiency gains in simulations based on higher-order perturbative impurity solvers. For instance, the fourth-order weak-coupling expansion or the OCA-level strong-coupling expansion scales as $N_{t}^{4}$. A truncation of the convolution integrals in these calculations reduces the computational effort to $O\left(N_{c}^{2} N_{t}^{2}\right)$. For $t_{\text {cut }} \ll t_{\text {max }}$, the truncation results in a significant speedup of the simulations and it is thus useful to explore under which conditions the errors incurred by the truncation remain negligible. For higher-order treatments, the cost of the evaluation of the self-energy can be reduced from $O\left(N_{t}^{n}\right)$ (with relatively large $n$ ) to $O\left(N_{t}^{2} N_{c}^{(n-2)}\right.$ ). In this context, it is interesting to note that a Monte Carlo based scheme such as the inchworm algorithm [79] automatically exploits these short memory times, which enables the sampling up to fairly high orders. In fact, the inchworm approach is based on the same stepwise time propagation and the same renormalized strong-coupling pseudoparticle propagators as the perturbative strong-coupling methods [49], and it would be interesting to compare the computational efficiency of the Monte Carlo sampling approach with that of a self-energy evaluation based on the truncation scheme introduced here. However, since the implementation of the perturbative strongcoupling diagrams beyond the third order requires a substantial coding effort, we leave this as an interesting topic for future investigations.

Another promising application of our scheme is its use within the Floquet implementation of DMFT [80-84], which directly treats the nonequilibrium steady state of periodically driven systems. These calculations include a coupling to a heat bath and interesting applications, such as the high-harmonic generation in solids [85], involve the simulation of highly excited nonequilibrium states. The memory times in these simulations can be expected to be short, and the cutoff scheme introduced here will enable more efficient simulations at low driving frequency, or the use of more accurate higher-order impurity solvers.

\section{ACKNOWLEDGMENTS}

This work was supported by the Swiss National Science Foundation through NCCR MARVEL and the European Research Council through ERC Consolidator Grant No. 724103. The calculations have been performed on the Beo04 cluster at the University of Fribourg, and the Piz Daint cluster at the Swiss National Supercomputing Centre (CSCS). 

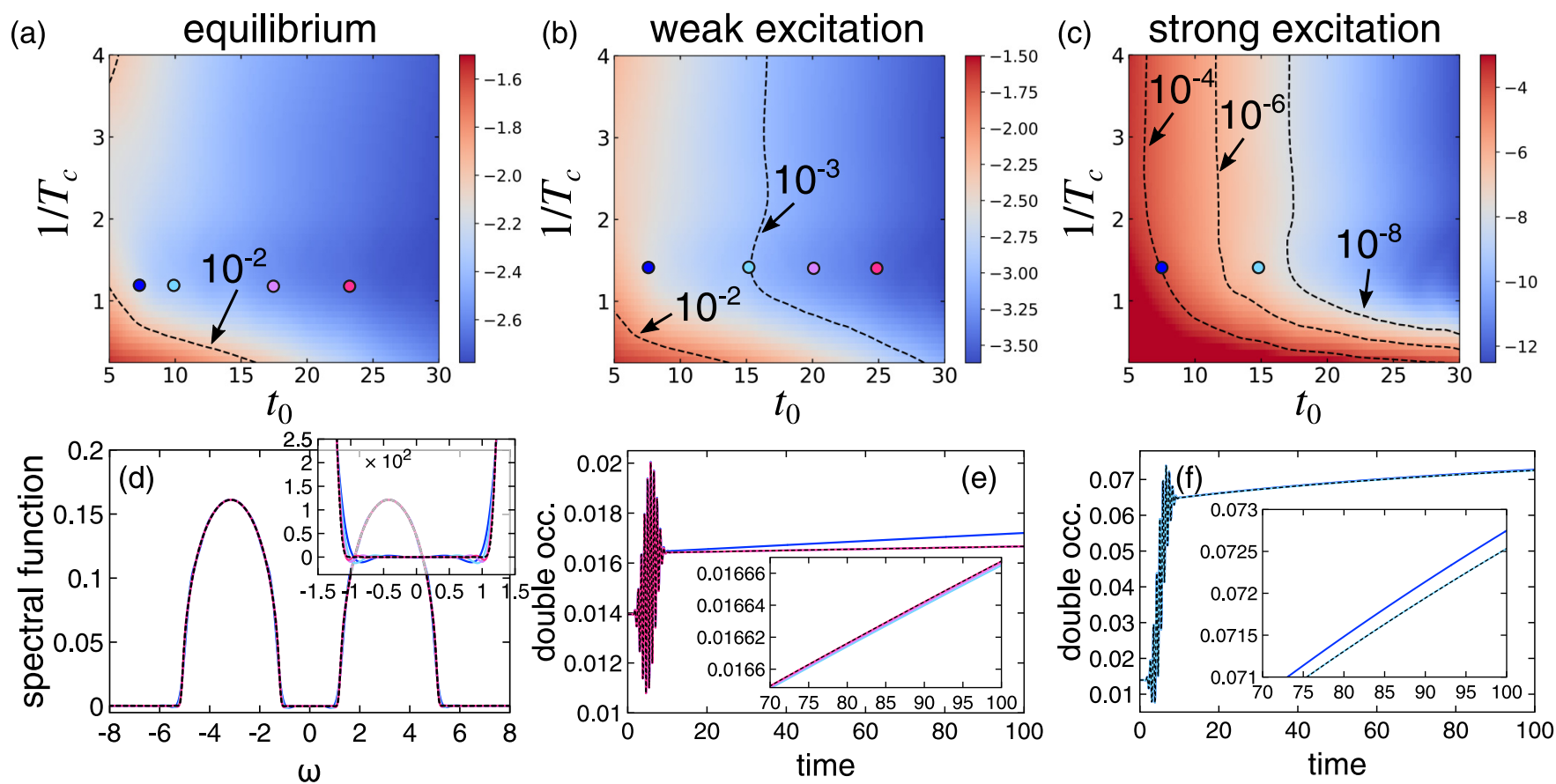

FIG. 14. Hubbard model in the paramagnetic phase. (a)-(c) Norm distance (logarithm) between the two-time reference GF (no cutoff) and $G_{\text {cut }}$ obtained by the cutoff scheme as a function of the memory time $t_{0}$ and sharpness of the cutoff $1 / T_{c}$ [cf. (9)] for $U_{0}=6$ and $\Delta U=0$ (a), $\Delta U=0.6$ (b), and $\Delta U=3.0$ (c). The contour lines delimit the regions where the error is smaller than the given values. The colored dots indicate representative values of $t_{0}$ and $T_{c}$, for which the equilibrium spectral function (d) and the double occupancy for weak (e) and strong excitation (f) are shown (consistent color coding). The black dashed lines represent the reference results.

\section{APPENDIX: RESULTS FOR LARGER GAP SIZE}

In this appendix, we present the norm error, spectral function, and time evolution of the double occupation or (a)
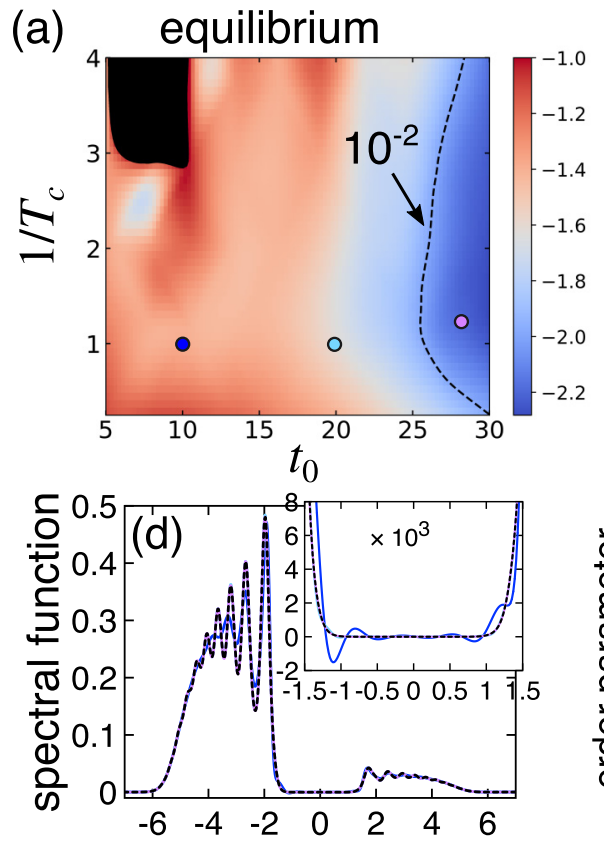

$\omega$ (b)
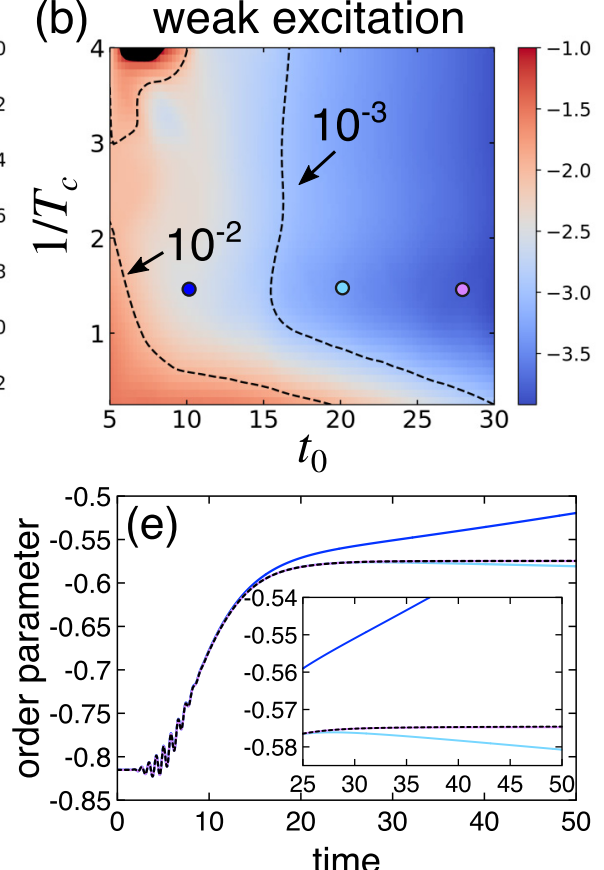

magnetization for the Hubbard and Holstein-Hubbard models with larger gap $\left(U_{0}=6\right)$ (Figs. 14-16). 
(a)
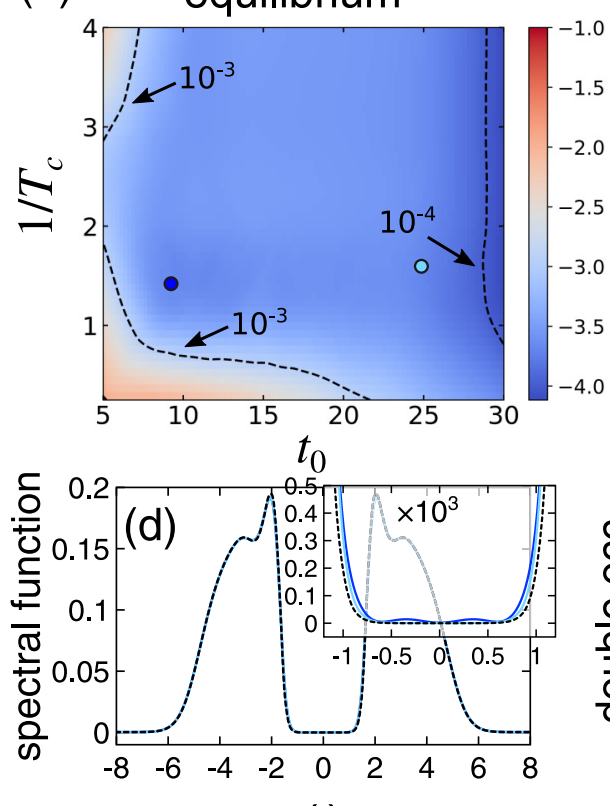

$\omega$ (b) weak excitation
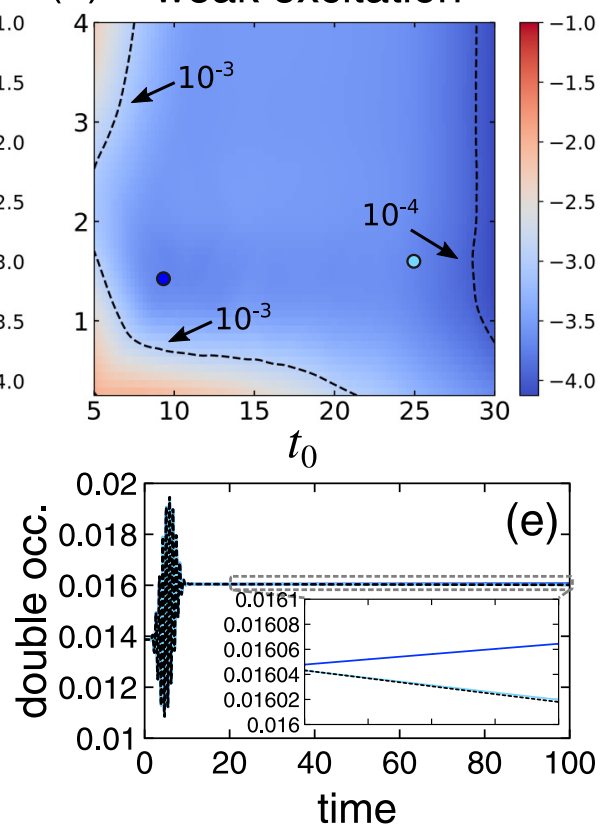

(c) strong excitation
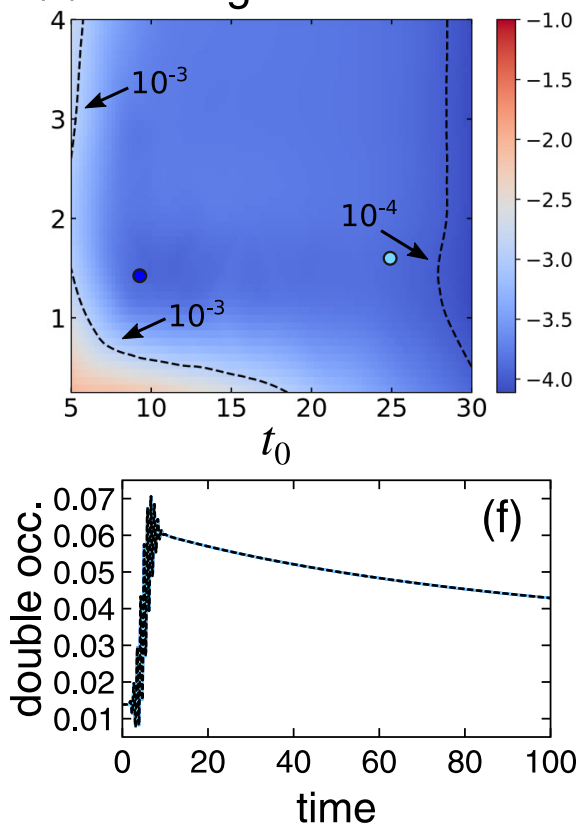

FIG. 16. Hubbard-Holstein model in the paramagnetic phase ( $\left.g=0.2, \omega_{0}=0.2\right)$. (a)-(c) Norm distance (logarithm) between the two-time reference GF $G_{\mathrm{loc}, \uparrow}\left(t, t^{\prime}\right)$ (no cutoff) and the GF obtained by the cutoff scheme for $U_{0}=6$ and $\Delta U=0$ (a), $\Delta U=0.6$ (b), and $\Delta U=3.0$ (c). (d) Equilibrium spin-up spectral function from the reference GF (black dashed line) and the cutoff scheme [colors as in (a)]. The dynamics of the double occupation is shown for $\Delta U=0.6$ (e) and $\Delta U=3.0$ (f).

[1] D. Fausti, R. I. Tobey, N. Dean, S. Kaiser, A. Dienst, M. C. Hoffmann, S. Pyon, T. Takayama, H. Takagi, and A. Cavalleri, Science 331, 189 (2011).

[2] S. Kaiser, C. R. Hunt, D. Nicoletti, W. Hu, I. Gierz, H. Y. Liu, M. Le Tacon, T. Loew, D. Haug, B. Keimer, and A. Cavalleri, Phys. Rev. B 89, 184516 (2014).

[3] L. Stojchevska, I. Vaskivskyi, T. Mertelj, P. Kusar, D. Svetin, S. Brazovskii, and D. Mihailovic, Science 344, 177 (2014).

[4] M. Mitrano, A. Cantaluppi, D. Nicoletti, S. Kaiser, A. Perucchi, S. Lupi, P. Di Pietro, D. Pontiroli, M. Riccò, S. R. Clark, D. Jaksch, and A. Cavalleri, Nature (London) 530, 461 (2016).

[5] S. Mor, M. Herzog, D. Golež, P. Werner, M. Eckstein, N. Katayama, M. Nohara, H. Takagi, T. Mizokawa, C. Monney, and J. Stähler, Phys. Rev. Lett. 119, 086401 (2017).

[6] A. L. Cavalieri, N. Müller, T. Uphues, V. S. Yakovlev, A. Baltuška, B. Horvath, B. Schmidt, L. Blümel, R. Holzwarth, S. Hendel, M. Drescher, U. Kleineberg, P. M. Echenique, R. Kienberger, F. Krausz, and U. Heinzmann, Nature (London) 449 , 1029 (2007).

[7] F. Krausz and M. Ivanov, Rev. Mod. Phys. 81, 163 (2009).

[8] J. Berges, S. Borsányi, and C. Wetterich, Phys. Rev. Lett. 93, 142002 (2004).

[9] M. Moeckel and S. Kehrein, Phys. Rev. Lett. 100, 175702 (2008).

[10] M. Eckstein, M. Kollar, and P. Werner, Phys. Rev. Lett. 103, 056403 (2009).

[11] M. Marcuzzi, J. Marino, A. Gambassi, and A. Silva, Phys. Rev. Lett. 111, 197203 (2013).

[12] L. D'Alessio, Y. Kafri, A. Polkovnikov, and M. Rigol, Adv. Phys. 65, 239 (2016).
[13] B. Bertini and M. Fagotti, J. Stat. Mech: Theory Exp. (2015) P07012.

[14] N. Tsuji, M. Eckstein, and P. Werner, Phys. Rev. Lett. 110, 136404 (2013).

[15] M. Schüler, Y. Murakami, and P. Werner, Phys. Rev. B 97, 155136 (2018).

[16] M. Kollar, F. A. Wolf, and M. Eckstein, Phys. Rev. B 84, 054304 (2011).

[17] M. Gring, M. Kuhnert, T. Langen, T. Kitagawa, B. Rauer, M. Schreitl, I. Mazets, D. A. Smith, E. Demler, and J. Schmiedmayer, Science 337, 1318 (2012).

[18] D. Golež, J. Bonča, L. Vidmar, and S. A. Trugman, Phys. Rev. Lett. 109, 236402 (2012).

[19] M. Sentef, A. F. Kemper, B. Moritz, J. K. Freericks, Z.-X. Shen, and T. P. Devereaux, Phys. Rev. X 3, 041033 (2013).

[20] P. Werner and M. Eckstein, Europhys. Lett. 109, 37002 (2015).

[21] Y. Murakami, P. Werner, N. Tsuji, and H. Aoki, Phys. Rev. B 91, 045128 (2015).

[22] H. Lu, S. Sota, H. Matsueda, J. Bonča, and T. Tohyama, Phys. Rev. Lett. 109, 197401 (2012).

[23] A. J. Daley, C. Kollath, U. Schollwöck, and G. Vidal, J. Stat. Mech: Theory Exp. (2004) P04005.

[24] K. Balzer and M. Bonitz, Nonequilibrium Green's Functions Approach to Inhomogeneous Systems (Springer, Berlin, 2012).

[25] G. Stefanucci and R. v. Leeuwen, Nonequilibrium Many-Body Theory of Quantum Systems: A Modern Introduction (Cambridge University Press, Cambridge, 2013).

[26] P. Lipavsky, V. Spicka, and B. Velicky, Phys. Rev. B 34, 6933 (1986). 
[27] G. Pal, Y. Pavlyukh, H. C. Schneider, and W. Hübner, Eur. Phys. J. B 70, 483 (2009).

[28] S. Latini, E. Perfetto, A.-M. Uimonen, R. van Leeuwen, and G. Stefanucci, Phys. Rev. B 89, 075306 (2014).

[29] K. Balzer, N. Schlünzen, and M. Bonitz, Phys. Rev. B 94, 245118 (2016).

[30] M. Galperin and S. Tretiak, J. Chem. Phys. 128, 124705 (2008)

[31] H. Ness and L. K. Dash, Phys. Rev. B 84, 235428 (2011).

[32] M. Bonitz, Quantum Kinetic Theory (Springer, Cham, 2015).

[33] T. Köhler, S. Rajpurohit, O. Schumann, S. Paeckel, F. R. A. Biebl, M. Sotoudeh, S. C. Kramer, P. E. Blöchl, S. Kehrein, and S. R. Manmana, Phys. Rev. B 97, 235120 (2018).

[34] H. Aoki, N. Tsuji, M. Eckstein, M. Kollar, T. Oka, and P. Werner, Rev. Mod. Phys. 86, 779 (2014).

[35] A. Georges, G. Kotliar, W. Krauth, and M. J. Rozenberg, Rev. Mod. Phys. 68, 13 (1996).

[36] J. K. Freericks, V. M. Turkowski, and V. Zlatić, Phys. Rev. Lett. 97, 266408 (2006).

[37] W. Metzner and D. Vollhardt, Phys. Rev. Lett. 62, 324 (1989).

[38] E. Müller-Hartmann, Z. Phys. B: Condens. Matter 74, 507 (1989).

[39] N. Tsuji, T. Oka, and H. Aoki, Phys. Rev. Lett. 103, 047403 (2009).

[40] M. Eckstein and P. Werner, Phys. Rev. B 84, 035122 (2011).

[41] P. Werner, K. Held, and M. Eckstein, Phys. Rev. B 90, 235102 (2014).

[42] T. Maier, M. Jarrell, T. Pruschke, and M. H. Hettler, Rev. Mod. Phys. 77, 1027 (2005).

[43] N. Tsuji, P. Barmettler, H. Aoki, and P. Werner, Phys. Rev. B 90, 075117 (2014).

[44] M. Eckstein and P. Werner, Sci. Rep. 6, 21235 (2016).

[45] R. Chitra and G. Kotliar, Phys. Rev. B 63, 115110 (2001).

[46] D. Golež, M. Eckstein, and P. Werner, Phys. Rev. B 92, 195123 (2015).

[47] F. Nilsson, L. Boehnke, P. Werner, and F. Aryasetiawan, Phys. Rev. Mater. 1, 043803 (2017).

[48] D. Golež, L. Boehnke, H. U. R. Strand, M. Eckstein, and P. Werner, Phys. Rev. Lett. 118, 246402 (2017).

[49] M. Eckstein and P. Werner, Phys. Rev. B 82, 115115 (2010).

[50] N. Tsuji and P. Werner, Phys. Rev. B 88, 165115 (2013).

[51] H.-P. Breuer and F. Petruccione, The Theory of Open Quantum Systems (Oxford University Press, Oxford, 2002).

[52] U. Weiss, Quantum Dissipative Systems (World Scientific, Singapore, 2012).

[53] H. Keiter and J. C. Kimball, J. Appl. Phys. 42, 1460 (1971).

[54] T. Pruschke and N. Grewe, Z. Phys. B: Condens. Matter 74, 439 (1989).

[55] A. Stan, N. E. Dahlen, and R. v. Leeuwen, J. Chem. Phys. 130, 224101 (2009).

[56] M. Schüler, J. Berakdar, and Y. Pavlyukh, Phys. Rev. B 93, 054303 (2016).
[57] N. Schlünzen and M. Bonitz, Contrib. Plasma Phys. 56, 5 (2016).

[58] M. Eckstein, T. Oka, and P. Werner, Phys. Rev. Lett. 105, 146404 (2010).

[59] R. Jördens, N. Strohmaier, K. Günter, H. Moritz, and T. Esslinger, Nature (London) 455, 204 (2008).

[60] C. Kollath, A. Iucci, I. P. McCulloch, and T. Giamarchi, Phys. Rev. A 74, 041604 (2006).

[61] F. Peronaci, M. Schiró, and O. Parcollet, Phys. Rev. Lett. 120, 197601 (2018).

[62] O. P. Matveev, A. M. Shvaika, T. P. Devereaux, and J. K. Freericks, Phys. Rev. B 94, 115167 (2016).

[63] D. Nicoletti and A. Cavalleri, Adv. Opt. Photonics 8, 401 (2016).

[64] T. F. Nova, A. Cartella, A. Cantaluppi, M. Först, D. Bossini, R. V. Mikhaylovskiy, A. V. Kimel, R. Merlin, and A. Cavalleri, Nat. Phys. 13, 132 (2017).

[65] Y. Pavlyukh, A. Rubio, and J. Berakdar, Phys. Rev. B 87, 205124 (2013).

[66] M. Schiró and M. Fabrizio, Phys. Rev. Lett. 105, 076401 (2010).

[67] M. Heyl, Phys. Rev. Lett. 113, 205701 (2014).

[68] B. Zunkovic, M. Heyl, M. Knap, and A. Silva, Phys. Rev. Lett. 120, 130601 (2018).

[69] P. Werner, N. Tsuji, and M. Eckstein, Phys. Rev. B 86, 205101 (2012).

[70] E. A. Yuzbashyan and M. Dzero, Phys. Rev. Lett. 96, 230404 (2006).

[71] R. A. Barankov and L. S. Levitov, Phys. Rev. Lett. 96, 230403 (2006).

[72] F. Peronaci, M. Schiró, and M. Capone, Phys. Rev. Lett. 115, 257001 (2015).

[73] G. Mazza, Phys. Rev. B 96, 205110 (2017).

[74] Y. Murakami, D. Golež, M. Eckstein, and P. Werner, Phys. Rev. Lett. 119, 247601 (2017).

[75] A. Lerose, J. Marino, B. Žunkovič, A. Gambassi, and A. Silva, Phys. Rev. Lett. 120, 130603 (2018).

[76] P. Werner and M. Eckstein, Phys. Rev. B 88, 165108 (2013).

[77] A. F. Kemper, M. A. Sentef, B. Moritz, J. K. Freericks, and T. P. Devereaux, Phys. Rev. B 90, 075126 (2014).

[78] K. Balzer and M. Eckstein, Phys. Rev. B 89, 035148 (2014).

[79] G. Cohen, E. Gull, D. R. Reichman, and A. J. Millis, Phys. Rev. Lett. 112, 146802 (2014).

[80] N. Tsuji, T. Oka, and H. Aoki, Phys. Rev. B 78, 235124 (2008).

[81] T. Mikami, S. Kitamura, K. Yasuda, N. Tsuji, T. Oka, and H. Aoki, Phys. Rev. B 93, 144307 (2016).

[82] Y. Murakami, N. Tsuji, M. Eckstein, and P. Werner, Phys. Rev. B 96, 045125 (2017).

[83] T. Qin and W. Hofstetter, Phys. Rev. B 96, 075134 (2017).

[84] M. E. Sorantin, A. Dorda, K. Held, and E. Arrigoni, Phys. Rev. B 97, 115113 (2018).

[85] Y. Murakami, M. Eckstein, and P. Werner, arXiv:1712.06460. 